\title{
A Phase-field/ALE Method for Simulating Fluid-Structure Interactions in Two-Phase Flow
}

\author{
X. Zheng ${ }^{\mathrm{a}}$, G.E. Karniadakis ${ }^{\mathrm{b}, *}$ \\ ${ }^{a}$ Department of Mechanical Engineering, Massachusetts Institute of Technology \\ ${ }^{b}$ Division of Applied Mathematics, Brown University
}

\begin{abstract}
We present a phase-field/ALE method for simulating fluid-structure interactions (FSI) in two-phase flow. We solve the Navier-Stokes equation coupled with the Cahn-Hilliard equation and the structure equation in an arbitrary Lagrangian Eulerian (ALE) framework. For the fluid solver, a spectral/hp element method is employed for spatial discretization and backward differentiation for time discretization. For the structure solver, a Galerkin method is used in Lagrangian coordinates for spatial discretization and the Newmark- $\beta$ scheme for time discretization. The mesh is updated from the initial configuration by a harmonic mapping constructed from the velocity of the interface between the fluid and the structure subdomains. To test the accuracy of the phase-field approach of this multi-physics method, we first simulate two-phase co-annular laminar flow in a stationary pipe and compare the results with the analytical solution. To test the accuracy of the FSI solver, we simulate a pipe conveying single-phase flow and compare the results with an existing validated code [1]. Finally, we present two numerical simulations of FSI in two-phase flow, specifically, in a flexible pipe conveying two fluids that induce self-sustained oscillations, and in external cross flow past a circular cylinder that modifies the classical vortex street due to a Kelvin-Helmholtz
\end{abstract}

\footnotetext{
*Corresponding author

Email address: george_karniadakis@brown.edu 401-863-1217 (G.E. Karniadakis)
} 
instability. These three-dimensional simulations demonstrate the capability of the method in dealing with FSI problems in two-phase flow with moving grids as well as its robustness and efficiency in handling different fluids with large contrast in physical properties.

Keywords:

Phase-field, ALE, FSI, pipe conveying fluid, Kelvin-Helmholtz instability, vortex street. 


\section{Introduction}

There are many applications of fluid-structure interactions (FSI) involving two-phase flow, for example, in pipes conveying gas or oil in industry $[2,3,4]$, vapour-liquid mixtures in heat exchangers [5, 6], air entrainment in hydraulic structures in civil engineering [7], and even blood flow through arteries in biological systems $[8,9]$. Among these, we focus here on a pipe conveying two different fluids, and on a pipe submerged in two different fluids, which may experience large vibrations that if not controlled properly they will cause severe damage to large-scale power systems such as pipe leaks, fatigue failures and even explosions [10]. There is already an extensive body of literature on FSI in single-phase flow that includes both experimental and numerical studies, documenting the relevant instabilities, i.e., buckling and flutter-like oscillations $[10,11]$. However, for FSI in two-phase flow so far most of the work has been focused on experiments, e.g., pipes conveying two fluids [3, 4, 12], and twophase external cross flow past a cylinder $[3,13,14,15,16,17]$. For pipes conveying two fluids, different flow regimes have been observed such as slug flow and churn flow [3, 18] but still the complex physics mechanisms behind the fluidelastic instabilities induced in flexible pipes have not been fully explained [18]. Moreover, other physical mechanisms associated with deformable interfaces between the fluid phases [19], as well as details of the interaction that occurs between hydrodynamic fluid forces and structure reacting forces [20] or induced structure vibrations have not been yet understood. Unlike the experimental work, there are very few reports on numerical simulations of FSI in two-phase flow and they mostly rely on drastically simplified models [18, 21]. In [21], a theoretical analysis of the effects of two-phase flow properties on the structure vibration based on a fluid-shell model is presented whereas 
in [18] a fluidelastic model assuming a simplified two-phase flow with uniform velocity is employed.

In order to accurately model FSI in two-phase flow, the challenge is to resolve the multiphysics emerging from the different properties of the fluids, the dynamics of the structure, and the distortions associated with the mesh movement while maintaining reasonable computational efficiency. In our approach, we adopt the phase-field formulation, the Galerkin method, and the ALE technique for the two-phase flow solver, structure solver, and mesh updating, respectively.

The phase-field approach $[22,23,24,25,26,27,28]$ enjoys several advantages in handling two-phase flow among other available methods, i.e., level-set, volume-of-fluids, or front tracking $[29,30,31,32,33,34]$. First, it is based on the minimization of the free energy and hence it can handle moving contact lines and deal with morphological changes of the interface. Second, the unified set of governing equations formulated over the entire domain can be solved on a fixed grid in a purely Eulerian fashion [22]. On the other hand, the computational complexity of the phase-field method increases as we have to deal with a fourth-order equation and variable properties throughout the domain. Here, we follow the algorithm in $[22,23]$ to deal with the system of Navier-Stokes and Cahn-Hilliard equations, which has the advantage of a fully decoupled system after discretization that can handle large fluid contrasts, e.g., density ratio, which is an important parameter for the dynamics of FSI in two-phase flow [21]. We employ the Galerkin method for the structure equation since it has good accuracy and can deal effectively with different boundary conditions by selecting appropriately the trial basis $[11,35]$. Here, the structure is modeled by the Euler-Bernoulli beam equation [11], taking into account both flexural and external tension effects. There are 
more general models for the structure, i.e., shell equation $[21,36]$, but the form considered here is sufficient for demonstrating the multi-physics coupling. Finally, we use the ALE technique to represent the interaction between the fluid and structure subdomains in our method. ALE, originally developed by [37, 38, 39], overcomes the shortcomings of purely Lagrangian and purely Eulerian descriptions and leads to accurate treatment of FSI [40]. Although ALE may be computationally more expensive [41] compared to other techniques, i.e., the coordinate transformation [1] or the use of non-conforming mesh with immersed boundary methods [41], it combines generality and accuracy that is required for FSI in two-phase systems.

The paper is organized as follows. In the next section we develop the numerical algorithm for solving the Navier-Stokes equation coupled with the Cahn-Hilliard equation and the structure equation in the ALE framework. In section 3, we test the accuracy of our method by simulating two-phase co-annular laminar flow in a stationary pipe and compare the results with the analytical solutions. Subsequently, we test the fluid and structure solvers and the interaction of the two by simulating a flexible pipe conveying single-phase flow and compare the results with those obtained from another validated code [1]. In section 4, we present two direct numerical simulations, first of a pipe conveying two-phase flow, and second of two-phase external cross flow past a circular cylinder. We conclude in section 5 with a short summary. The appendices include details of each algorithm and values of all the parameters used in the numerical tests. 


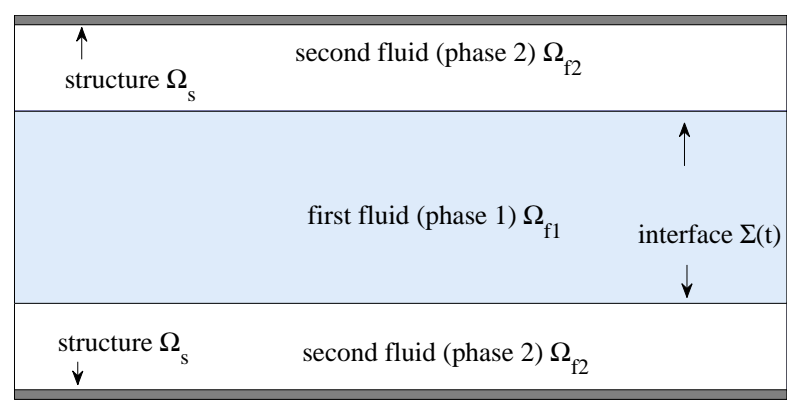

(a) Internal

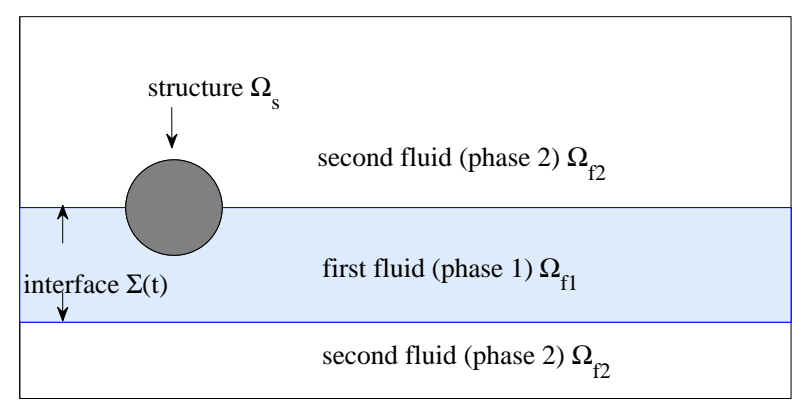

(b) External

Figure 1: Sketch of problems of interest (a) internal flow with the first fluid surrounded by the second (b) two-phase external cross flow past a circular cylinder.

\section{Numerical methods and computational framework}

\subsection{Problem setup}

In FSI problems, the domain $\Omega \subset R^{3}$ is composed of two parts: the subdomain $\Omega_{f}$ occupied by the fluid, and the subdomain $\Omega_{s}$ occupied by a compliant or deformable structure, as shown in Fig. 1. The fluid subdomain $\Omega_{f}$ contains two fluids, i.e., the first fluid (phase 1) and the second fluid (phase 2). There is a common boundary between the two subdomains, which is the fluid-structure interface $\sum(\mathrm{t})=\Omega_{f} \bigcap \Omega_{s}$. The domain $\Omega$ is moving with the movement of the interface $\sum(\mathrm{t})$.

The fluid model, characterized by the Navier-Stokes and Cahn-Hilliard equations, is stated in the ALE framework [42]. The structure model, governed by the Euler-Bernoulli beam equation (including tension) for internal flow or the elastically mounted cylinder motion equation for external cross flow, is stated in a purely Lagrangian approach. There are four sets of variables in this system: the fluid velocity $\mathbf{u}(\mathbf{x}, \mathrm{t})$, the mesh velocity $\mathbf{w}(\mathbf{x}, \mathrm{t})$, the structure displacement $\boldsymbol{\eta}(\mathbf{x}, \mathrm{t})$ and the phase-field variable $\boldsymbol{\phi}(\mathbf{x}, \mathrm{t})$. Here $\mathbf{x}$ and $\mathbf{X}$ are the 
position vectors in the deformed configuration and initial configuration, respectively, while s denotes the Lagrangian coordinate. A complete list of all symbols is presented in Table 1.

\subsection{Mathematical formulation and physical model}

We present all the governing equations next for the multi-physics problem we consider. Two-phase fluid model:

$$
\begin{aligned}
& \rho\left(\frac{\partial \mathbf{u}}{\partial t}+(\mathbf{u}-\mathbf{w}) \cdot \nabla \mathbf{u}\right)=-\nabla p+\nabla \cdot\left[\mu\left(\nabla \mathbf{u}+\nabla \mathbf{u}^{T}\right)\right]-\lambda \nabla \cdot(\nabla \phi \nabla \phi)+\mathbf{f}(\mathbf{x}, t) \\
& \nabla \cdot \mathbf{u}=0 \\
& \frac{\partial \phi}{\partial t}+(\mathbf{u}-\mathbf{w}) \cdot \nabla \phi=-\lambda \gamma_{1} \nabla^{2}\left[\nabla^{2} \phi-h(\phi)\right]+g(\mathbf{x}, t), \\
& \nabla^{2} \mathbf{w}=0, \text { on } \Omega_{f} \\
& \mathbf{w}=\frac{\partial \eta}{\partial t}, \text { on } \sum(t)
\end{aligned}
$$

Among the set of Eqs. (1a)-(1e), (1a) is the Navier-Stokes equation, (1b) is the incompressibility condition on the velocity, (1c) is the Cahn-Hilliard equation and (1d) is the harmonic mapping from the initial configuration to the current configuration. Eq. (1e) enforces the continuity of the velocities on the interface $\sum(t)$ across the fluid and structure subdomains. Dirichlet or Neumann boundary conditions are imposed in the fluid subdomain $\Omega_{f}$. Dynamic contact angle boundary conditions are imposed for the phase-field variable $\phi(\mathbf{x}, \mathrm{t})[23]$; for simplicity, here we use a contact angle $90^{\circ}$. Boundary and initial conditions for Eqs. (1a) -(1c) are given as in [22] and for Eqs. (1d)-(1e) as in [43]; see also Appendix A. We employ the spectral element method in space and backwards differentiation formulas (BDF) in time to discretize the Navier-Stokes and Cahn-Hilliard equations on the fluid subdomain $\Omega_{f}$, i.e., Eqs. (1a)-(1c). The discretized formulations for Eqs. (1a)-(1c) are given as 
in [22] and for Eqs. (1d)-(1e) as in [43]; see also Appendix B.

\section{Structure model:}

Let $\eta(\mathbf{s}, t)=\left(q_{x}, q_{y}, q_{z}\right)$ represent the structure displacements, where $q_{x}, q_{y}$ and $q_{z}$ denote out-of-plane, vertical and axial displacements, repectively. In this paper, in order to simplify the presentation of the results we assume that the pipe vibrates only in vertical (y) direction with $q_{x}, q_{z}=0$. This is typically the case for low Reynolds number but at high Reynolds number and for a certain parameter range, out-of-plane motion and even axial oscillations may arise. The motion of the structure is given by the mixed beam-elastically mounted cylinder equation:

$$
m_{s} \frac{\partial^{2} q_{y}}{\partial t^{2}}+E I \frac{\partial^{4} q_{y}}{\partial s^{4}}-T \frac{\partial^{2} q_{y}}{\partial s^{2}}=F_{y}
$$

which simplifies to an elastically mounted cylinder equation if we model the vibration through a spring-mass-damper system, i.e.,

$$
m_{s} \frac{\partial^{2} q_{y}}{\partial t^{2}}+k q_{y}=F_{y}
$$

For Eq. 2, we assume that both ends of the slender structure with length L are simply supported and apply the following boundary conditions:

$$
\left.q_{y}\right|_{z=0}=0,\left.\quad \frac{\partial^{2} q_{y}}{\partial s^{2}}\right|_{z=0}=0,\left.\quad q_{y}\right|_{z=L}=0,\left.\quad \frac{\partial^{2} q_{y}}{\partial s^{2}}\right|_{z=L}=0
$$

For Eq. 2, we employ the Galerkin method [11] for discretization in space, using as basis functions the eigenfunctions of a beam, $\phi_{k}(s, t)$, for the vertical displacement. The displacement can be represented as a series of the basis functions:

$$
q_{y}(s, t)=\sum^{N} q_{y_{k}}(t) \phi_{k}(s, t)
$$


where $\mathrm{N}$ is the total number of the eigenmodes, $\phi_{k}(t)=\sin (k \pi s)$ are the basis functions, and $q_{y_{k}}(t)$ are unknown functions of time to be determined. The resulting ordinary differential equations in matrix form are:

$$
M_{i j} \ddot{q}_{y j}+K_{i j} q_{y j}=Q_{y}
$$

where $M$ and $K$ are the mass and stiffness coefficient matrices resulted from the linear terms in the vertical direction, and $Q_{y}$ are the resulting matrices from discretizing the fluid force term $F_{y}$, see Appendix C. The size of this set of ordinary differential equations depends on the number of Galerkin modes $N$ used in the discretization.

For Eq. 2 and Eq. 3, we use the Newmark- $\beta$ scheme with $\beta=0.25$ for time discretization.

\subsection{Algorithm Implementation}

Instead of following the monolithic approach and solve for all state variables simultaneously (an approach that may not easily scale up to many processors), we instead employ a partitioned algorithm, whereby in each iteration step, the fluid and structure solvers are solved separately and interact by exchanging suitable transmission conditions at the interface $\sum(t)$, as sketched in Fig. 2. In summary, at the $n$-th time step, we solve the FSI system using the following algorithm:

1. (Solid) Solve the structure Eq. (5) with hydrodynamic fluid forces applied by the fluid, then update the structure solver results $((\eta),(\dot{\eta}),(\ddot{\eta}))$.

2. (Interface) Pass the velocity $\dot{\eta}$ at the interface from structure to fluid.

3. (Fluid) Solve the Navier-Stokes and Cahn-Hilliard Eqs. (1a)-(1c) and calculate the hydrodynamic forces $\left(F_{\{y, t\}}=\int\left(\left[-p^{n} \mathbf{I}+\mu\left(\nabla \mathbf{u}^{n}+\left(\nabla \mathbf{u}^{n}\right)^{T}\right)\right] \mathbf{n}_{f}\right) d s\right)$, where the integration 
is performed around the circumference of the beam at each spanwise location and $\mathbf{n}_{f}$ is the normal vector of fluid subdomain pointing outward on the interface $\sum(t)[1]$.

4. (Mesh) Update the mesh velocity boundary condition at the interface with $\mathbf{w}^{n}=\dot{\boldsymbol{\eta}}^{n}$.

5. (Mesh) Obtain the mesh velocity $\mathbf{w}^{n}$ by solving (1d).

6. (Mesh) Update the mesh positions for the fluid subdomain using numerical integration, i.e.,

$$
\frac{\mathbf{x}^{n}-\sum_{i=1}^{J} \hat{\alpha}_{i} \mathbf{x}^{n-i}}{\Delta t}=\sum_{i=1}^{J} \hat{\hat{\alpha}}_{i} \mathbf{w}^{n-i}
$$

Here $\hat{\alpha}_{i}$ and $\hat{\hat{\alpha}}_{i}$ are the coefficients for the corresponding time integration schemes, as in [44]; details about the mesh updating can be found in [43].

7. Go to time step $n+1$.

The partitioned algorithm is stable even for low mass ratios by applying the technique of fictitious added mass or fictitious pressure $[45,46]$. The fluid and structure interaction requires passing the hydrodynamic fluid force to structure and updating the structure

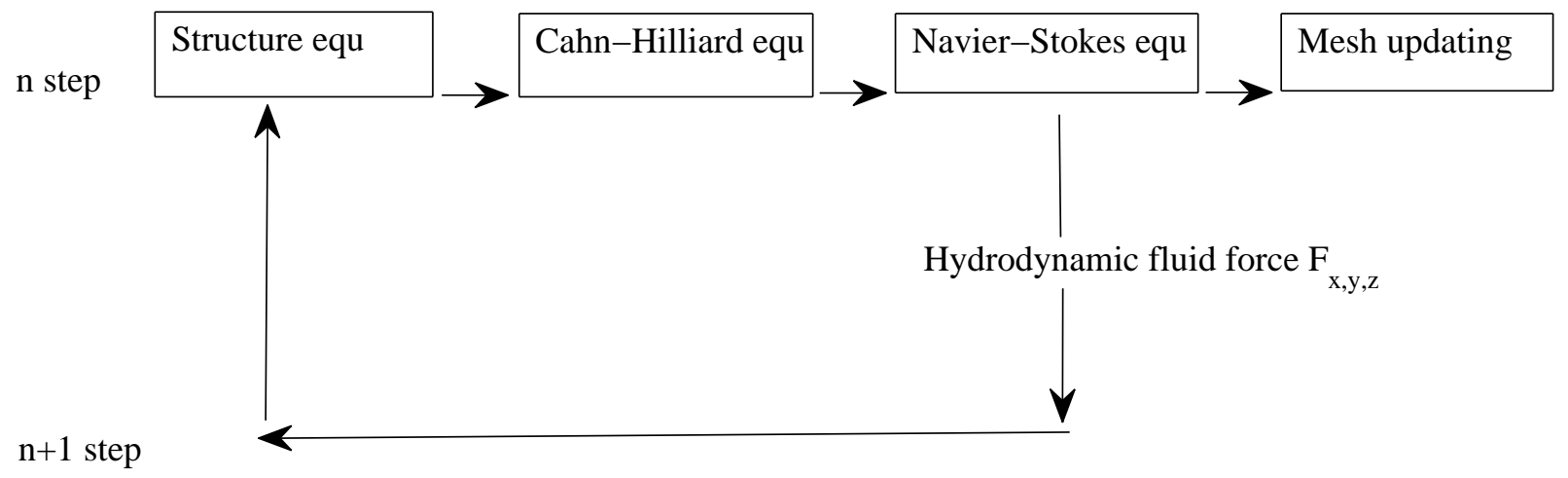

Figure 2: FSI coupling procedure at time step n. $F_{x, y, z}$ stands for the hydrodynamic fluid force acting upon the slender structure at the interface. 
displacement and velocity every time step. In the following, we show how we calculate the hydrodynamic fluid forces $F_{y}$ acting on the interface $\Sigma(\mathrm{t})$, where $F_{\{y, t\}}=\int\left(\left[-p^{n} \mathbf{I}+\mu\left(\nabla \mathbf{u}^{n}+\right.\right.\right.$ $\left.\left.\left.\left(\nabla \mathbf{u}^{n}\right)^{T}\right)\right] \mathbf{n}_{f}\right) d s$. Here $p$ is pressure, $\left.\rho_{(} \phi\right)=\frac{\left(\rho_{1}+\rho_{2}\right)}{2}+\frac{\left(\rho_{1}-\rho_{2}\right)}{2} \phi$ is the fluid density, and $n_{f}$ is the normal vector at the interface.

1. Calculate $\mu=\frac{\left(\mu_{1}+\mu_{2}\right)}{2}+\frac{\left(\mu_{1}-\mu_{2}\right)}{2} \phi$. (Note: $\mu$ is the dynamic viscosity)

2. Calculate the $\mathbf{n}_{f}$ on interface $\Sigma(\mathrm{t})$.

3. Obtain the values of $\mathbf{u}, \mathrm{p}, \mu$ and $\mathbf{n}_{f}$ on the quadrature points at the interface $\Sigma(\mathrm{t})$.

4. Obtain the values of $\mathbf{u}, \mathrm{p}, \mu$ and $\mathbf{n}_{f}$ on the line around the circumference of the beam, where the integration will be performed.

5. Calculate the line integration $F_{\{y, t\}}=\int\left(\left[-p^{n} \mathbf{I}+\mu\left(\nabla \mathbf{u}^{n}+\left(\nabla \mathbf{u}^{n}\right)^{T}\right)\right] \mathbf{n}_{f}\right) d s$ around the circumference of the beam at each spanwise location.

\section{Numerical simulations}

In this section, we first demonstrate the accuracy of the phase-field method by simulating two-phase co-annular laminar flow in a stationary pipe. Subsequently, we demonstate the accuracy of the ALE framework by simulating a pipe conveying single-phase flow and comparing the results with those from the code Nektar2.5d-Fourier [1], which solves the Navier-Stokes equations by a spectral element method in $x-y$ plane and Fourier discretization along the $\mathrm{z}$ direction with coordinates attached on the structure instead of tracking the mesh as in the ALE technique. Finally, we simulate pipes conveying two-phase flow and also two-phase external cross flow past a circular cylinder to show the capability of our method in handling FSI problems for both internal and external two-phase flows. 
To facilitate subsequent discussions, non-dimensional flow variables and physical parameters used are shown in Appendix D.

\subsection{Numerical simulations I-Accuracy verification}
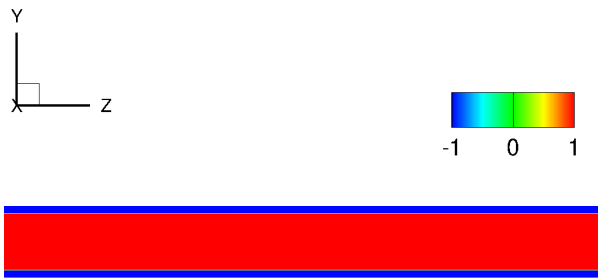

(a)

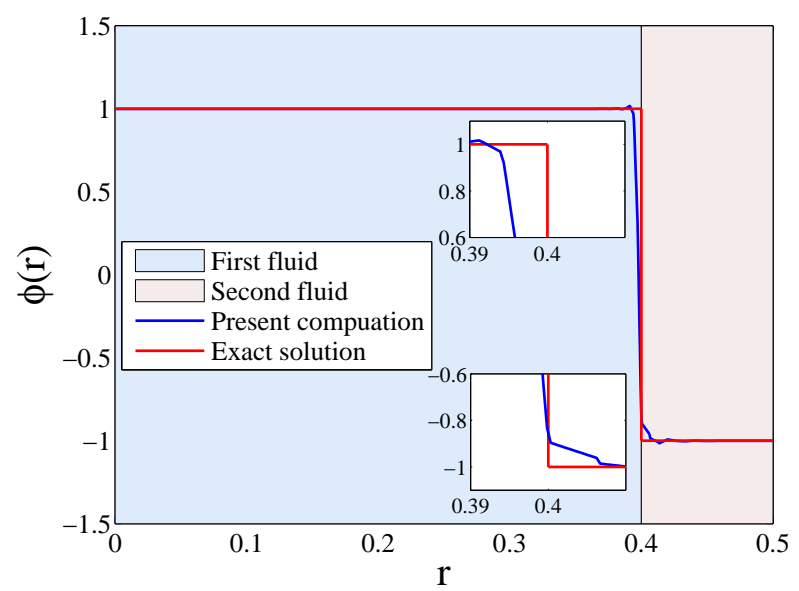

(c) (b)

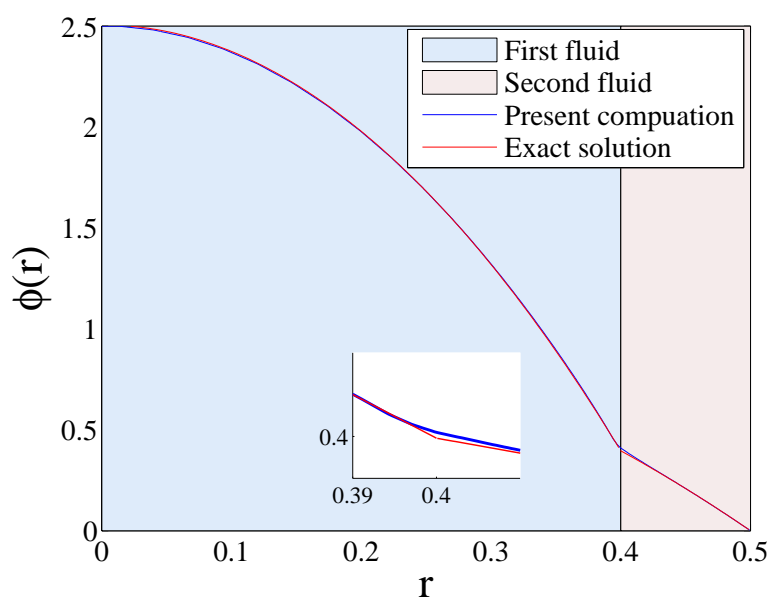

(d)

Figure 3: Two-phase co-annular laminar flow in a stationary pipe. Density ratio $=50.0$. Dynamic viscosity ratio $=10.0$. Interface thickness $=0.001$. (a) and (c) show the phase-field contours and profiles (zoomed in at the interface of two fluids) in steady state $(t=30)$; (b) and (d) show axial velocity contours and profiles (zoomed in at the interface of two fluids) in steady state.

\subsubsection{Verification example 1: Two-phase co-annular laminar flow in a stationary pipe}

In this section, we test our method by simulating two-phase co-annular immiscible laminar flow in a stationary pipe. The sketch of the problem of interest is shown in Fig. 1(a). By 
co-annular we refer to two fluids in the pipe with one fluid surrounded by the other. The effect of gravity is neglected. Although the flow is axisymmetric at these Reynolds numbers, we employ a fully 3D discretization to test our solvers. An element of polynomial order 3 has been used for all the elements. A time step size $\Delta t=10^{-3}$ has been used in the simulation. We use 9150 hexahedra to discretize the 3D domain with 305 elements in $\mathrm{x}-\mathrm{y}$ plane and 30 layers along the $\mathrm{z}$ (axial) direction. Details about boundary and initial conditions for the fluid and the phase-field variables $(\mathbf{u}, \boldsymbol{\phi})$ are given in Appendix E, which are the same as in [47]. Table 2 shows the parameters we used, namely a density ratio of $\rho_{2} / \rho_{1}=50.0$ and dynamic viscosity ratio of $\mu_{2} / \mu_{1}=10.0$. We also used surface tension and interface mobility of $10^{-3}$ in non-dimensional units magnititude. Fig. 3(c) shows that the phase-field profile from the simulation achieves good agreement with the exact co-annular phase-field profile. Fig. 3(d) shows that the velocity profile from the simulation overlaps with that from the analytical solution. The simulation results of this section demonstrate that our algorithm can be an accurate method for simulating two-phase co-annular laminar flow in a stationary pipe with large density ratios. It allows the accurate calculation of two-phase flow with a thin interface thickness as small as $10^{-3}$.

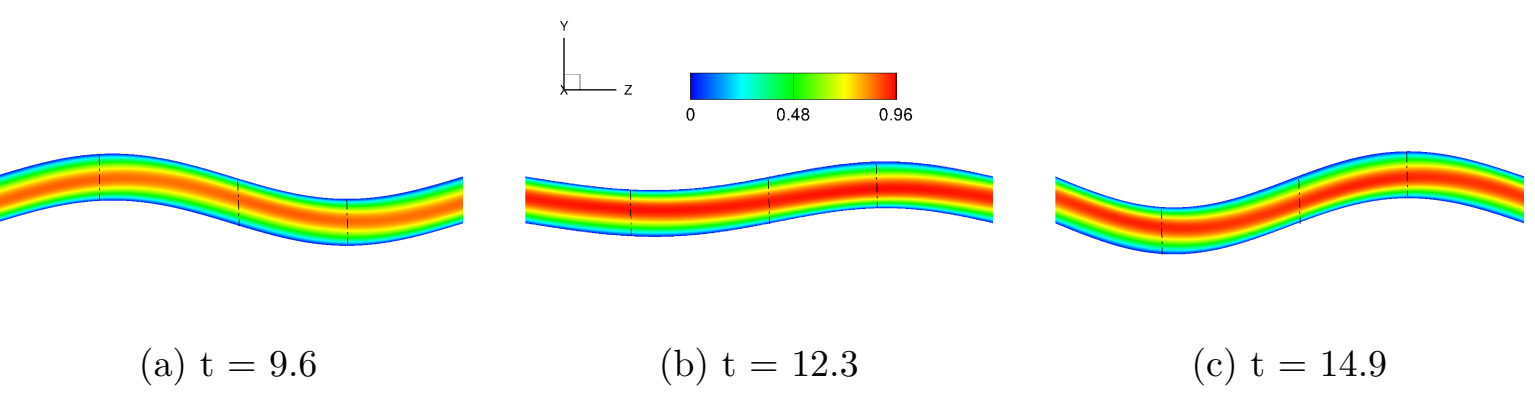

Figure 4: Pipe conveying single-phase flow - axial velocity contours on $y-z$ plane at $\mathrm{x}=0$ (a) $\mathrm{t}=9.6$, (b) $\mathrm{t}=12.3,(\mathrm{c}) \mathrm{t}=14.9$. Dashdot line: $\mathrm{z}=2.3,5,7.1$. 


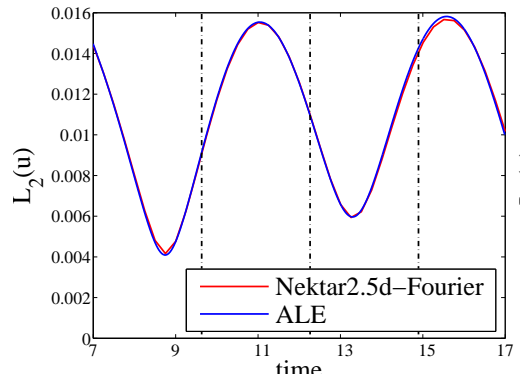

(a) $L_{2}$ norm of $u$

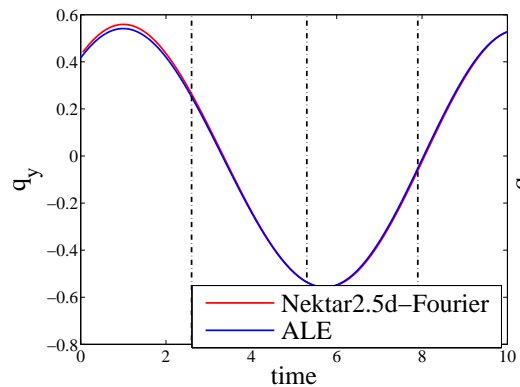

(d) Displacement at $\mathrm{z}=2.3$

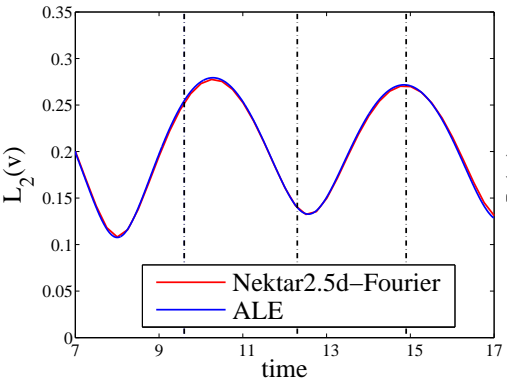

(b) $L_{2}$ norm of $\mathrm{v}$

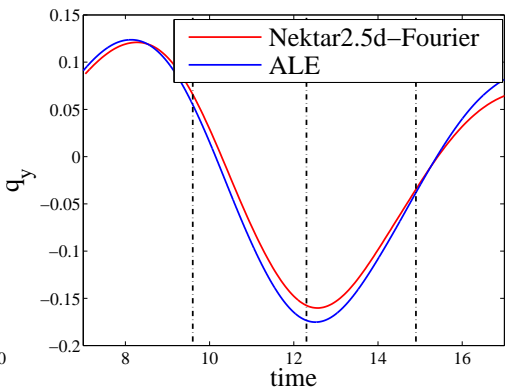

(e) Displacement at $\mathrm{z}=5$

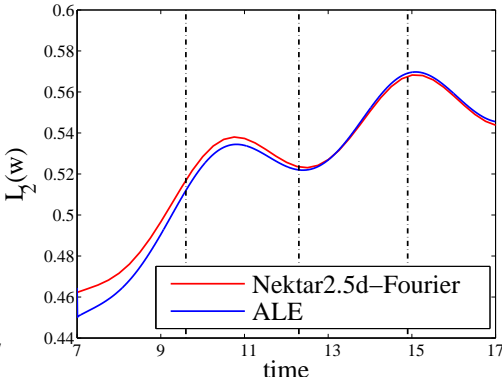

(c) $L_{2}$ norm of w

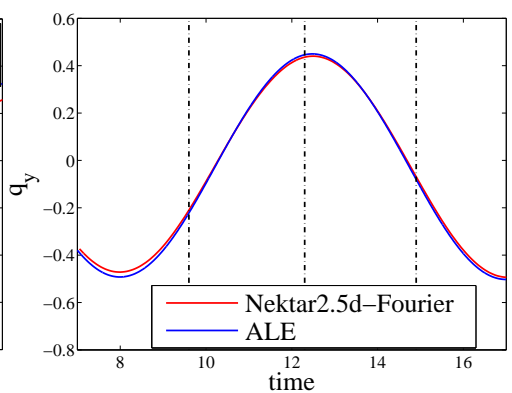

(f) Displacement at $\mathrm{z}=7.1$

Figure 5: $1^{\text {st }}$ row: $L_{2}$ norm of flow velocity $\mathbf{u}(\mathrm{u}, \mathrm{v}, \mathrm{w})$ for pipe conveying single-phase flow at $R e=50.2^{\text {nd }}$ row: vertical displacement time history at $\mathrm{z}=2.3, \mathrm{z}=5$ and $\mathrm{z}=7.1$. Red line: Nektar2.5d-Fourier code ; Blue line: present computation ; Dotted line: $\mathrm{t}=9.6, \mathrm{t}=12.3$ and $\mathrm{t}=14.9$.

\subsubsection{Verification example 2: Pipe conveying single-phase flow}

In this section, we simulate a flexible pipe conveying single-phase flow. See Appendix F for boundary conditions. We first force the pipe to vibrate in sinunoidal motion up to time $\mathrm{t}=7$ and then let the pipe vibrate freely under the perturbed flow. Then, we compare both fluid and structure profiles from our method with those from the Nektar2.5d-Fourier code [1]. The parameters are given in Table 3. Here the $L_{2}$ norm of $\mathbf{u}(u, v, w)$ is used as the characteristic velocity $U_{0}$ and $I_{p}$ is a non-dimensional parameter indicating instability for the pipe system. See Table 1 and Appendix D for definitions.

The pipe is modeled by the EulerBernoulli beam equation with both ends simply sup- 
ported, see Eqs. 2 and 4. For the velocity, we use periodic boundry conditions in z (axial) direction and no-slip boundary conditions on the pipe walls.

Fig. 4 shows axial velocity contours at time $t=9.6,12.3$ and 14.9 from our method. Figs. 5(a)-(c) show the $L_{2}$ norm of the flow velocity, and Figs. 5(d)-(f) show the vertical displacement at $\mathrm{z}=2.3,5$ and 7.1 of the pipe from our method and Nektar2.5d-Fourier code. The well-matched results demonstrate the accuracy of our method in simulating a flexible pipe conveying a fluid and subject to flow-induced vibrations.

\subsection{Numerical simulations II-pipe conveying two-phase flow}

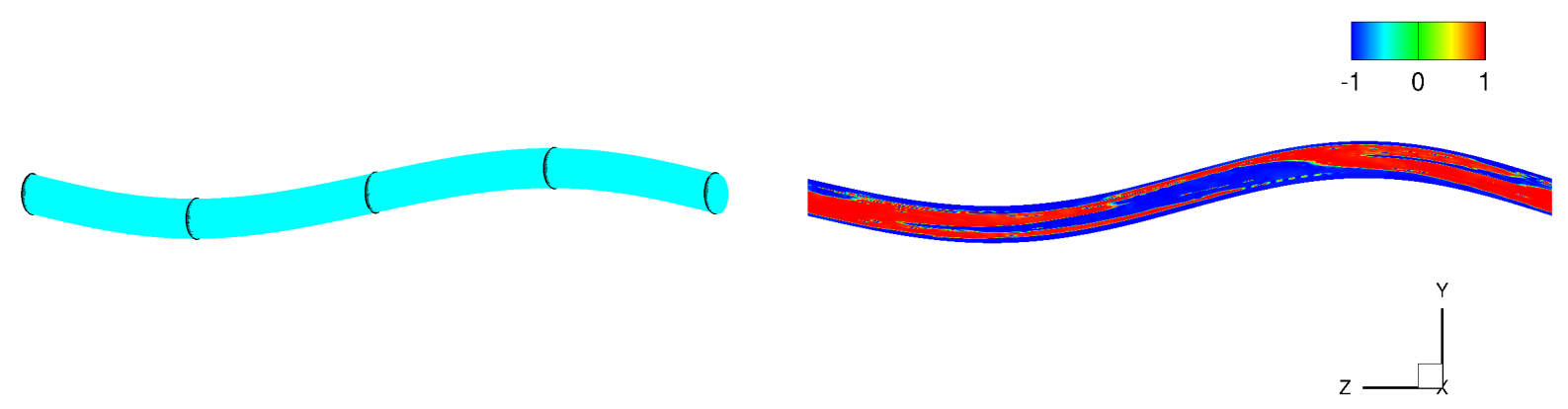
(a) pipe
(b) phase-field plot

Figure 6: Sketch of problem of interest-pipe conveying two-phase flow. (a) pipe vibration (b) phase-field showing two fluids at $\mathrm{y}-\mathrm{z}$ (centerline) plane $(\mathrm{x}=0)$. Red color: first fluid; Blue color: second fluid.

In this subsection, we simulate pipes conveying two-phase flow. The sketch of the problem of interest is shown in Fig. 6. Specifically, we investigate the dynamics of two pipe systems, which have the same density ratio 8.0, dynamic viscosity ratio 1.0 and void fraction 0.6 but different Reynolds number $R e$ and tension related parameter $I_{p}$. Detailed initial and boundary conditions for the velocity and the phase-field variables $(\mathbf{u}, \boldsymbol{\phi})$ are given in Appendix F. We first forced the pipe to vibrate till 60s for about 10 time periods and then the 
pipe is allowed to vibrate freely in the y direction. More specifically, we use diameter D and length $\mathrm{L}=20 \mathrm{D}(\mathrm{D}=1$ is the characteristic length) for the pipe, which occupies the domain $\Omega=\{(\mathrm{r}, \mathrm{z}):-0.5 \leq \mathrm{r} \leq 0.5,0 \leq \mathrm{z} \leq 20\}$, where $\mathrm{r}$ is the radius of the cross section. To simulate this problem, we discretize the domain with 48060 hexahedron elements, with 801 elements in the $\mathrm{x}-\mathrm{y}$ plane and 60 layers along the $\mathrm{z}$ (axial) direction. Interpolation with polynomial order 3 has been used for all the elements. A time step size $\Delta t$ is set at $10^{-3}$. Here we use a full three-dimensional formulation and not an axi-symmetric formulation which is not general.

Fig. 7(a) and Fig. 7(b) show that a stable flow and vorticity patterns are developed for the pipe system with $\left(R e, I_{p}\right)=(257,0.05)$. From the contours of the axial velocity and $\mathrm{x}$-vorticity on the $\mathrm{x}-\mathrm{y}$ plane at $\mathrm{z}=5,10$ and 15 , we see that the axial velocity and $\mathrm{x}$-vorticity are symmetric with respect to the x-axis. Fig. $7(\mathrm{c})$ shows that the annular twophase flow pattern remains almost the same until the pipe stops vibrating. Fig. $7(\mathrm{~d})$ shows that the amplititude of the pipe vibration in the y direction is decaying with time. The FFT analysis in Fig. 7(f) shows that the dominant (non-dimensional) frequency of the vertical displacement of the pipe is about 0.12 .

Fig. 8(a) and Fig. 8(b) show that an unstable flow and vorticity patterns are developed for the pipe system with $\left(R e, I_{p}\right)=(931,0.92)$. From the contours of the axial velocity and $\mathrm{x}$-vorticity on the $\mathrm{x}-\mathrm{y}$ plane at $\mathrm{z}=5,10$ and 15 , we see that the axial velocity and $\mathrm{x}$-vorticity are asymmetric with respect to the x-axis. Fig. 8(c) shows that the annular two-phase flow pattern changes to a flow with large bubbles at about $t=150$. Fig. $8(d)$ shows that the amplititude of the pipe vibration in the y direction is approaching a constant value. The FFT analysis in Fig. 8(f) shows that the dominating frequency is about 0.025. From Fig. 7 


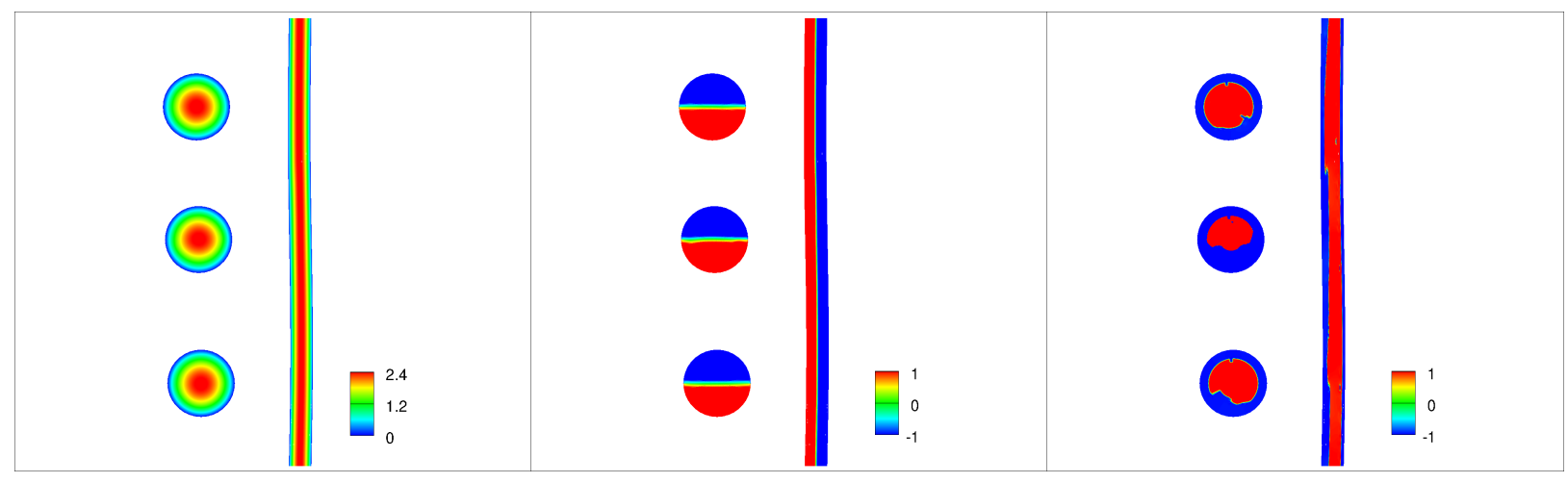

(a) axial velocity

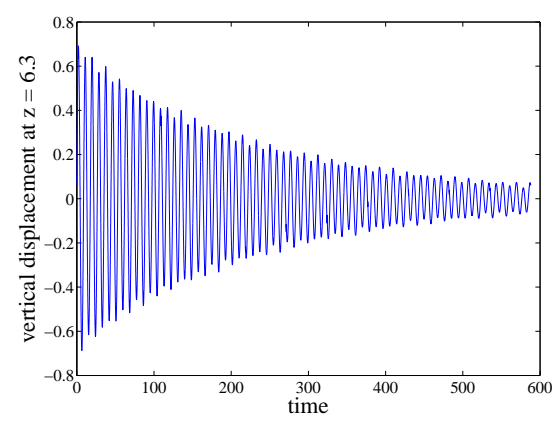

(d) vertical displacement (b) x-vorticity

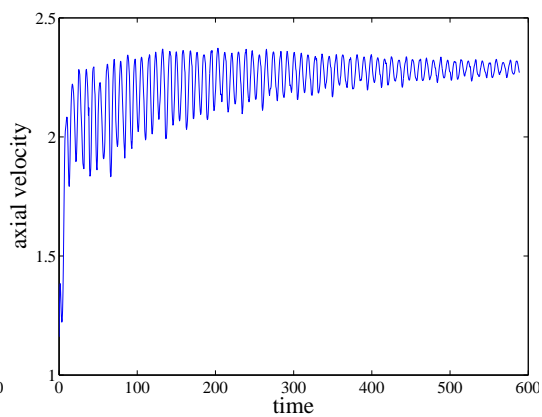

(e) time history of axial velocity (c) phase-field

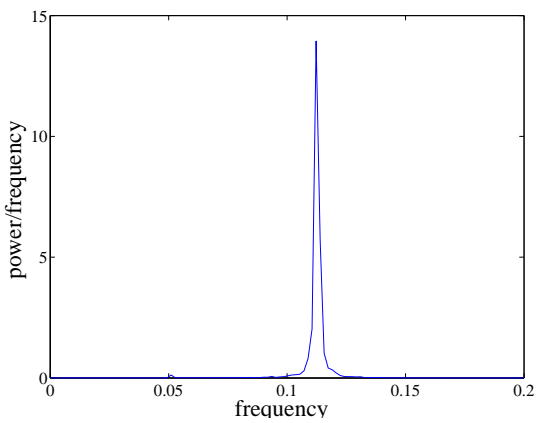

(f) PSD of displacement

Figure 7: Case 1. Internal two-phase flow at $\left(R e, I_{p}\right)=(257,0.05) .1^{\text {st }}$ row: axial velocity, x-vorticity and phase-field contour profile at $\mathrm{y}-\mathrm{z}$ plane $(\mathrm{x}=0)$ and $\mathrm{x}-\mathrm{y}$ plane $(\mathrm{z}=5,10$ and 15$) ; 2^{\text {nd }}$ row: vertical displacement at $\mathrm{z}=6.3$, time history of axial velocity at point $(0.1,0.1,5)$ and power spectral density (PSD) analysis of displacement time history at $\mathrm{z}=6.3$. Frequency is identified as $f=\frac{n^{2} \pi \sqrt{E I /\left(m+m_{f}\right)}}{2 L^{2}} \sqrt{\left(1+\frac{T L^{2}}{E I \pi^{2} n^{2}}\right)}$.

and Fig. 8, we see that with the same void fraction and density ratio, but higher $\left(R e, I_{p}\right)$, the amplititude of the pipe vibration in the y direction is larger and the frequency is smaller. For $\left(R e, I_{p}\right)=(931,0.92)$, the pipe system exhibits self-sustained vibrations.

\subsection{Numerical simulations III-two-phase external cross flow past a circular cylinder}

In this section, we simulate external single-phase and two-phase flow past a stationary and a freely vibrating circular cylinder. The goal is to investigate vortex shedding patterns and differences of the amplititude of a freely vibrating cylinder between single-phase and two- 
phase flow. The cylinder is modeled by the elastically mounted cylinder motion equation, see Eq. 3. We consider a cylinder in a domain with length 40D and height 20D (D = 1 is the characteristic length). It occupies the domain $\Omega=\{(\mathrm{x}, \mathrm{y}, \mathrm{z}):-10 \leq \mathrm{x} \leq 30,-10 \leq \mathrm{y} \leq$ $10,0 \leq \mathrm{z} \leq 0.2\}$. Detailed initial and boundary conditions for the velocity and the phasefield variables $(\mathbf{u}, \phi)$ are given in Appendix G. The effect of gravity is neglected, and the characteristic velocity $U_{0}=1.0$, which is also the inflow velocity. Initially, the first fluid occupies the domain from $\mathrm{y}=\left[\begin{array}{ll}-3 & 0\end{array}\right]$ while the second fluid occupies the region $\mathrm{y}=\left[\begin{array}{ll}-10 & -3\end{array}\right]$ and $y=\left[\begin{array}{ll}0 & 10\end{array}\right]$. At the very beginning, half of the cylinder is immersed in the first fluid and the other half is in the second fluid; see Fig. 1(b). Table 5 gives the parameters used in our simulation. Periodic boundaries are imposed along the $\mathrm{y}$ and $\mathrm{z}$ directions, and inflow (Dirichlet) and outflow (Neumann) boundary conditions are imposed in the $\mathrm{x}$ direction. 
Table 1

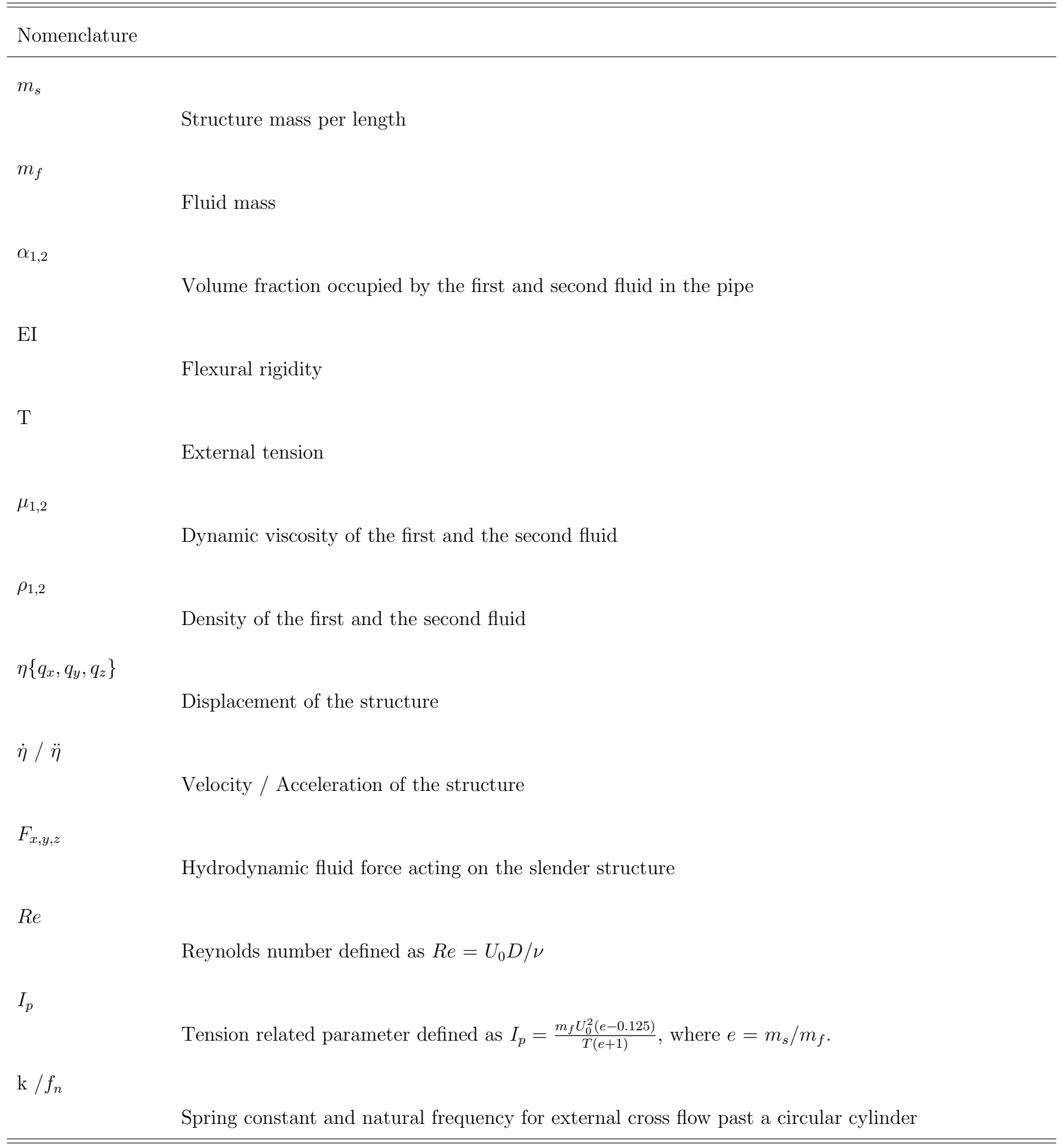


Table 2: Parameters used for two-phase co-annular laminar flow in a stationary pipe with length 10D, where $\mathrm{D}$ is the pipe diameter.

\begin{tabular}{lllc}
\hline \hline & Density & Dynamic Viscosity & $R e$ \\
\hline First fluid & 1.0 & 0.01 & 100 \\
Second fluid & 50.0 & 0.1 & 500 \\
\hline \hline
\end{tabular}

Table 3: Parameters used for pipe conveying single-phase flow with length 10D, where D is the pipe diameter.

\begin{tabular}{cccccccc}
\hline \hline Parameters & $m_{s}$ & $\mathrm{~T}$ & $\mathrm{EI}$ & $\mu$ & $L_{2}(w)$ & $R e$ & $I_{p}$ \\
\hline case 1 & 6 & 6 & 6 & 0.01 & 0.5 & 50 & 0.028 \\
\hline \hline
\end{tabular}

Table 4: Parameters used for pipe conveying two-phase flow with length 20D.

\begin{tabular}{|c|c|c|c|c|c|c|c|c|c|c|c|}
\hline Parameters & $m_{s}$ & $\alpha_{1}$ & $\rho_{1}$ & $\rho_{2} / \rho_{1}$ & $\mathrm{~T}$ & EI & $\mu_{1}$ & $\mu_{2} / \mu_{1}$ & $L_{2}(w)$ & $\mathrm{Re}$ & $I_{p}$ \\
\hline case 1 & 20 & 0.6 & 1 & 8 & 96.8 & 300 & 0.02 & 1 & 1.35 & 257 & 0.05 \\
\hline case 2 & 20 & 0.6 & 1 & 8 & 16 & 300 & 0.01 & 1 & 2.45 & 931 & 0.92 \\
\hline
\end{tabular}

Table 5: Parameters used for flow past a circular cylinder in a duct.

\begin{tabular}{ccccc}
\hline \hline Parameters & $m_{s}$ & Density ratio & Dynamic viscosity ratio & $R e$ \\
\hline single-phase & 6 & & 100 \\
two-phase & 6 & 3.0 & 1.0 & 100 \\
\hline \hline
\end{tabular}




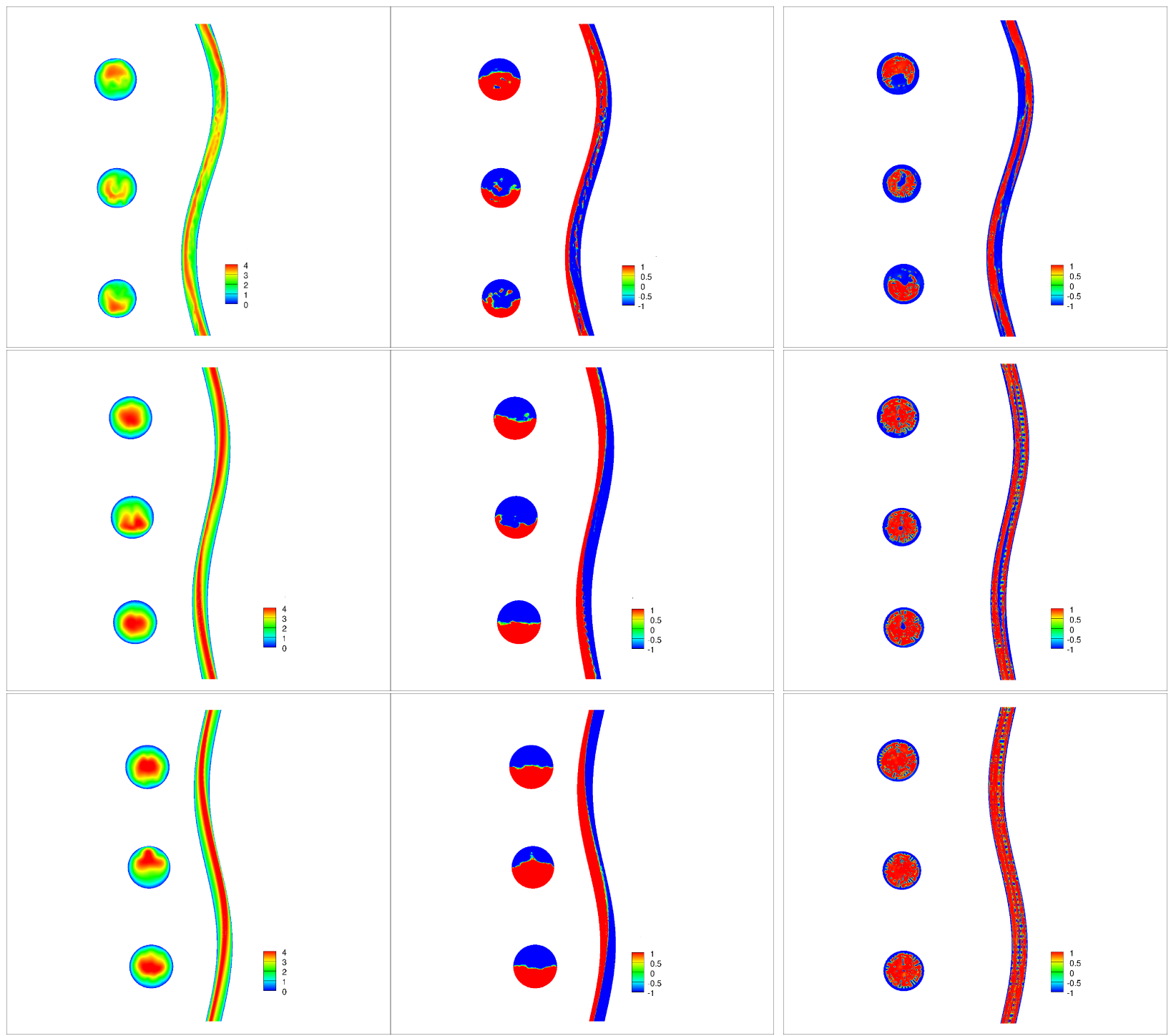

(a) axial velocity

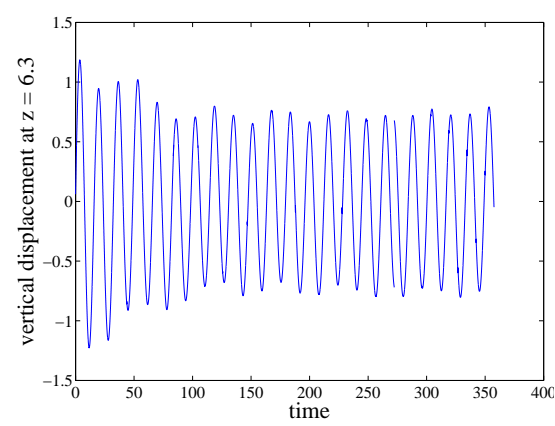

(d) vertical displacement (b) x-vorticity

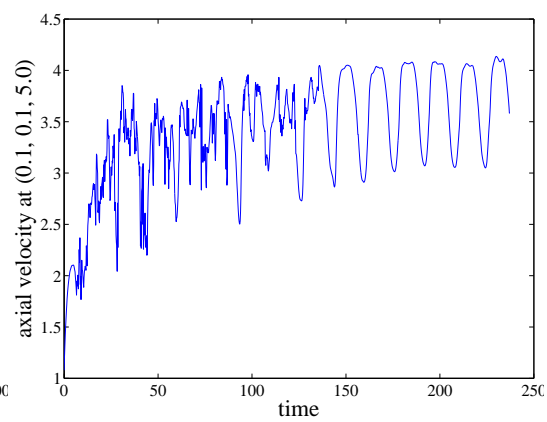

(e) time history of axial velocity (c) phase-field

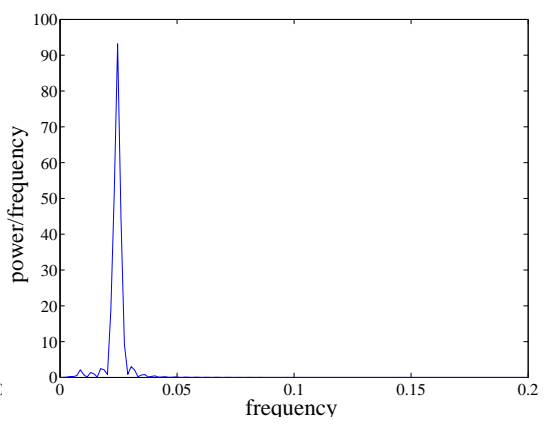

(f) PSD of displacement

Figure 8: Case 2. Internal two-phase flow at $\left(R e, I_{p}\right)=(931,0.92) .1^{s t}-3^{r d}$ row: axial velocity, x-vorticity and phase-field contours at $\mathrm{y}-\mathrm{z}$ plane $(\mathrm{x}=0)$ and $\mathrm{x}-\mathrm{y}$ plane $(\mathrm{z}=5,10,15) .1^{\text {st }}-3^{\text {rd }}$ row: $\mathrm{t}=43,150,282$; $4^{\text {th }}$ row: vertical displacement at $\mathrm{z}=6.3$, time histom of axial velocity at point $(0.1,0.1,5.0)$ and PSD of displacement time history at $\mathrm{z}=6.3$. 


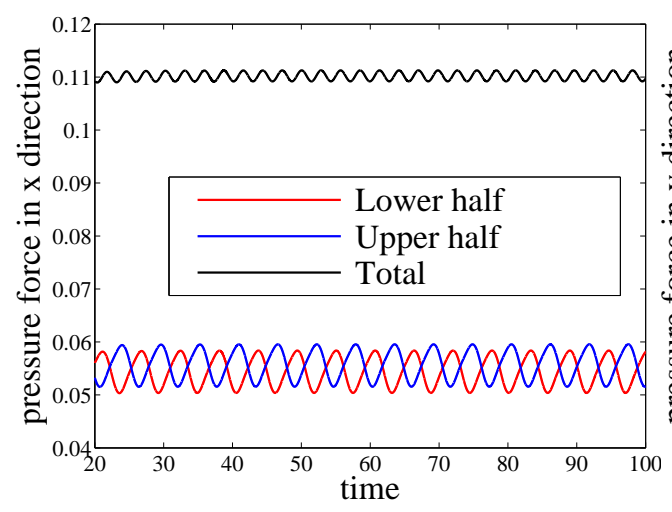

(a) single-phase $\mathrm{x}$ direction

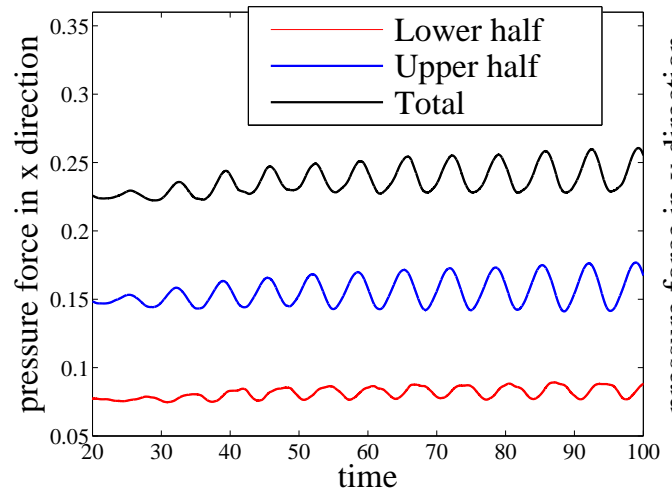

(c) two-phase $\mathrm{x}$ direction

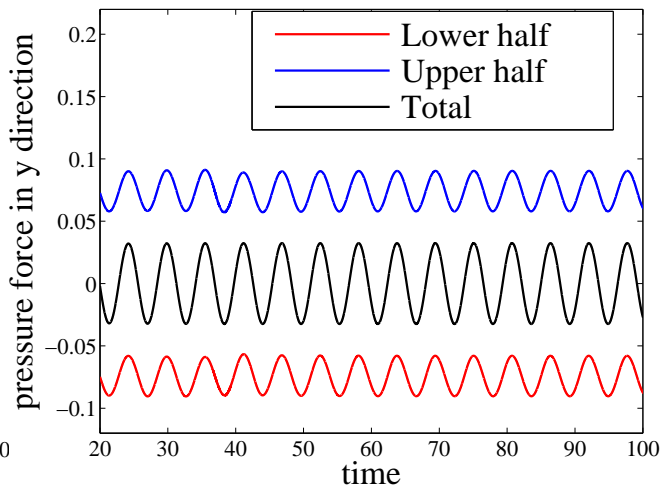

(b) single-phase y direction

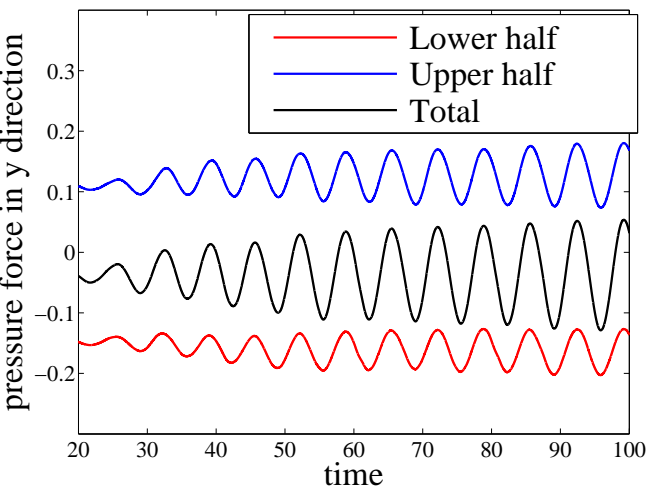

(d) two-phase y direction

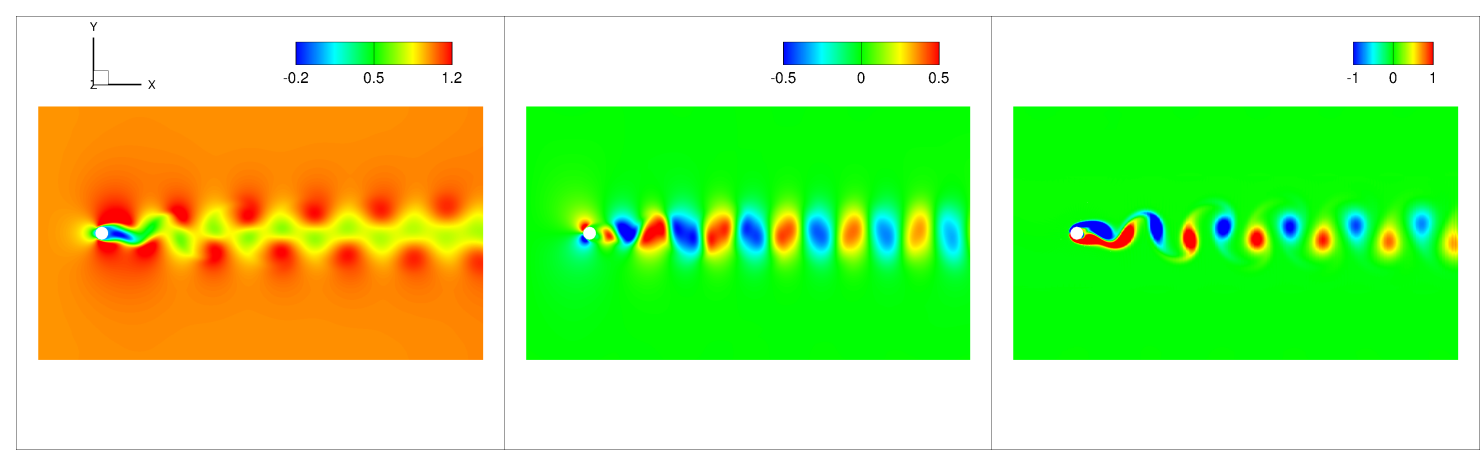

(e) single-phase u-velocity

(f) single-phase v-velocity

(g) single-phase z-vorticity

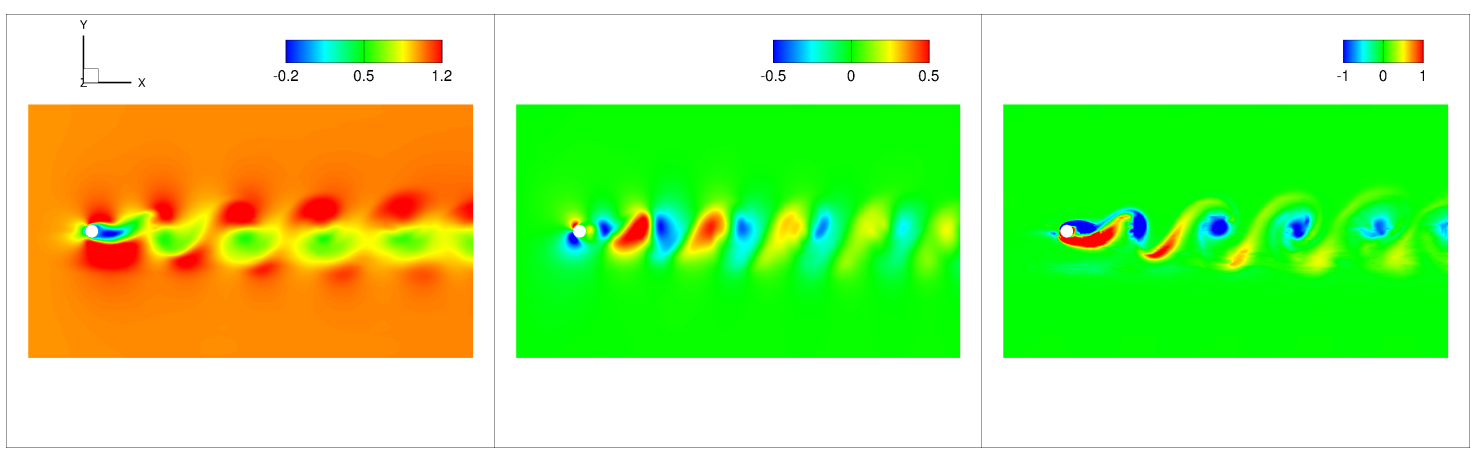

(h) two-phase u-velocity

(i) two-phase v-velocity

(j) two-phase z-vorticity

Figure 9: Stationary cylinder with $R e=100$ at $\mathrm{t}=100.1^{\text {st }}$ and $2^{\text {nd }}$ row: pressure forces in the $\mathrm{x}$ and $\mathrm{y}$ directions; $3^{\text {rd }}$ and $4^{\text {th }}$ row: velocity and vorticity contours. 


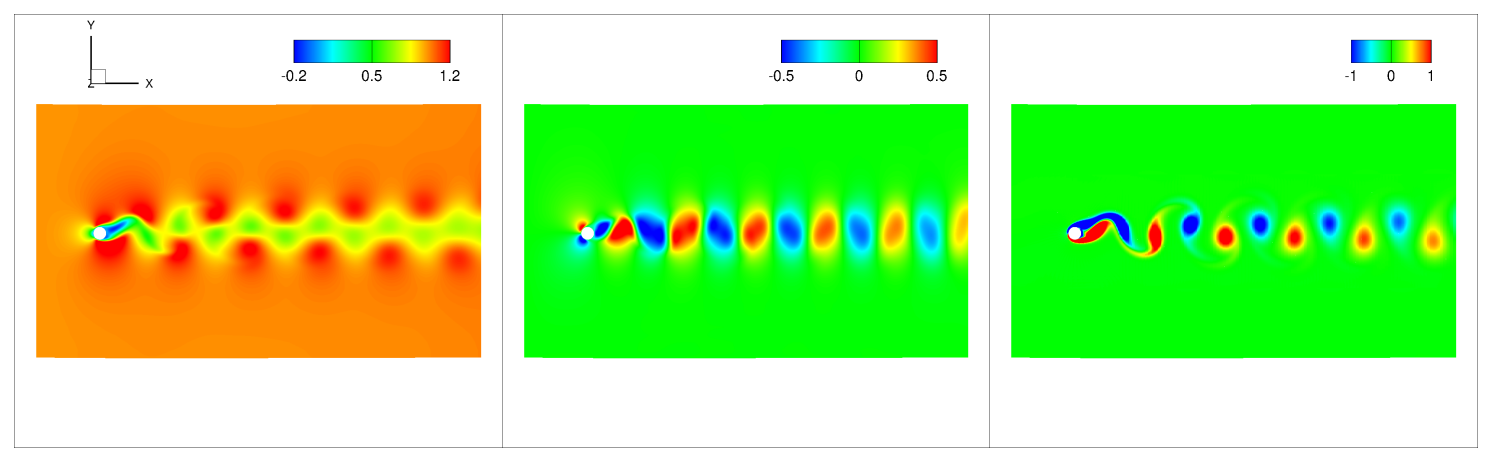

(a) single-phase u-velocity

(b) single-phase v-velocity

(c) single-phase z-vorticity

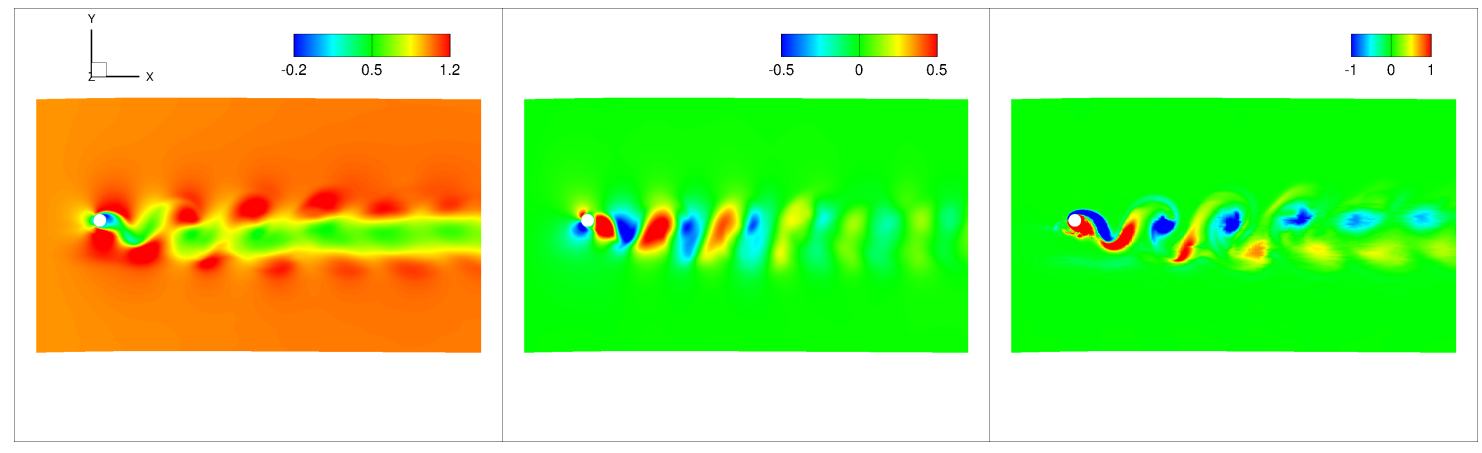

(d) two-phase u-velocity

(e) two-phase v-velocity

(f) two-phase z-vorticity

Figure 10: Free cylinder with $\left(R e, f_{n}\right)=(100,0.167)$ at $\mathrm{t}=100$. Contour plot of velocity in the $\mathrm{x}$ and $\mathrm{y}$ directions and vorticity in $\mathrm{z}$ direction. $1^{\text {st }}$ row: single-phase ; $2^{\text {nd }}$ row: two-phase.
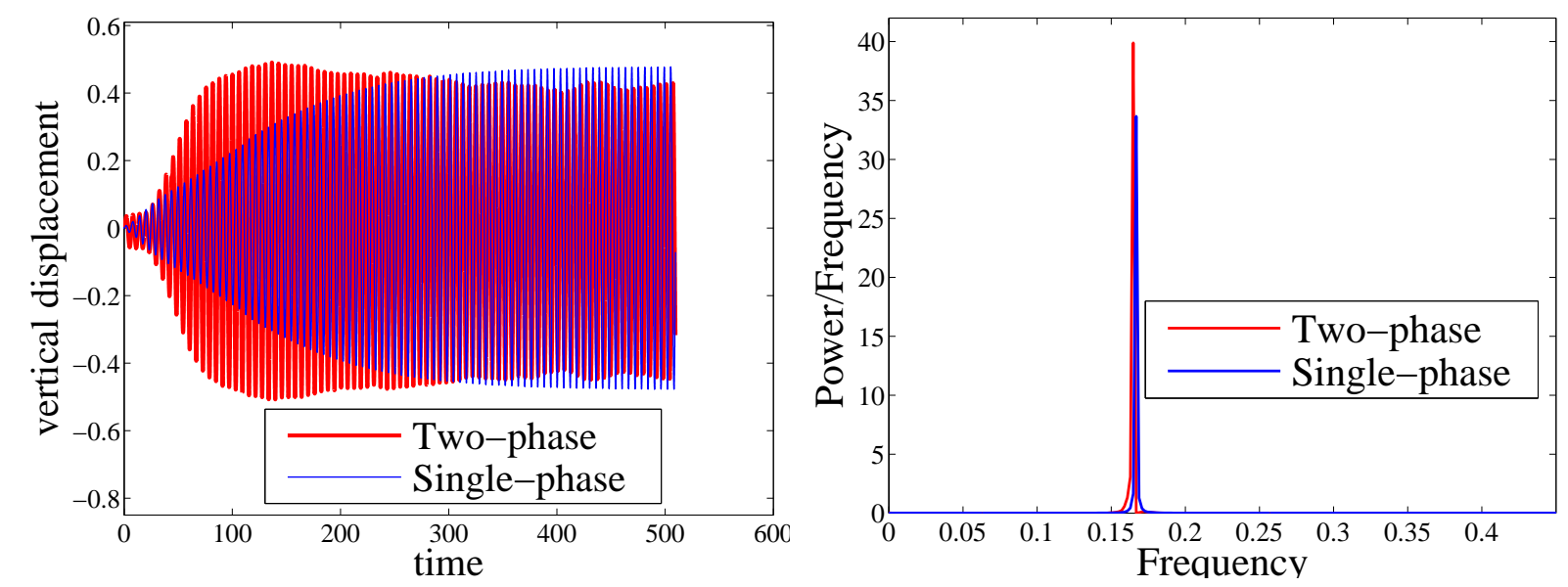

Figure 11: Free cylinder vertical displacement time history with $\left(R e, f_{n}\right)=(100,0.167)$. (a) vertical displacement (b) PSD analysis of vertical displacement. 
We first consider the stationary cylinder case. We start by comparing the hydrodynamic fluid forces acting on the cylinder between single-phase and two-phase flow. Figs. 9(a)-(b) show the hydrodynamic fluid forces acting on the cylinder in the $\mathrm{x}$ and $\mathrm{y}$ directions in singlephase flow. In the $\mathrm{x}$ direction, the forces on the upper and lower half of the cylinder are the same but there is about a half-period difference in phase. In the y direction, the absolute value of the force on the upper and lower half of the cylinder are almost the same. The force on the whole cylinder in the $\mathrm{y}$ direction is symmetric with respect to the line $\mathrm{y}=0$. Figs. 9(c)-(d) show hydrodynamic fluid forces acting on the cylinder in the $\mathrm{x}$ and $\mathrm{y}$ directions in two-phase flow. In the $\mathrm{x}$ direction, the force on the upper half is larger than that on the lower half. In the y direction, the absolute value of the force on the upper half is smaller than that on the lower half. Therefore, the force on the whole cylinder in the y direction is no longer symmetric with respect to the line $\mathrm{y}=0$ but symmetric with respect to the line $\mathrm{y}=-0.12$ instead. The difference of the force distribution between single-phase and two-phase flow is due to the fluid density difference on the upper and lower half of the cylinder. Fig. 9(a) and Fig. 9(c) show that in the $\mathrm{x}$ direction the fluid force on the lower half of the cylinder is almost the same for single-phase and two-phase flow but significantly different on the upper half of the cylinder. Fig. 9(b) and Fig. 9(d) show that in the y direction the forces on both the upper half and lower half in two-phase flow are larger than their counterparts in single-phase flow.

Next, we look at the vortex shedding patterns for the stationary cylinder in single-phase and two-phase flow. Fig. $9(\mathrm{~g})$ shows that for the single-phase case with $R e=100$, vortices are shed alternatively from the upper and lower surfaces of the cylinder, creating a periodic flow pattern. However, Fig. 9(j) shows that for two-phase case, vortices shed from the 
lower surface of the cylinder are larger than those from the upper surface, and that the negative vorticty is surrounded by the positive vorticity. Fig. 10 compares the u- and vvelocity and z-vorticity fields between single-phase and two-phase flow past a freely vibrating circular cylinder with $\left(R e, f_{n}\right)=(100,0.167)$. From Fig. 10(a)-(c), we can see symmetric patterns for the velocity and the vorticity for single-phase flow. However, for two-phase flow, both velocity and vorticity patterns lose their symmetry along the line $\mathrm{y}=0$ as shown in Fig. 10(d)-(f). Fig. 11 shows that the displacements in single-phase flow are symmetric with respect to the origin and exhibit a lock-in phenomenon. For two-phase flow, the displacement is asymmetric with respect to the origin. Up to time $t=300$ s, the displacement in two-phase flow is larger than that in the single-phase flow, and then it decreases to be smaller than that in the single-phase flow. The PSD analysis shows that single-phase and two-phase flow past a circular cylinder have the same frequency. Fig. 12 shows the snapshots of the phasefield and z-vorticity contours at different times. Figs. 12(a)-(c) show the formation of a Kelvin-Helmholtz instability on the interface of the two fluids with different densities. Figs. 12(d)-(f) show the vorticity patterns. Figs. 12(g)-(i) show that the z-vorticity contours are consistent with the phase-field contours. 


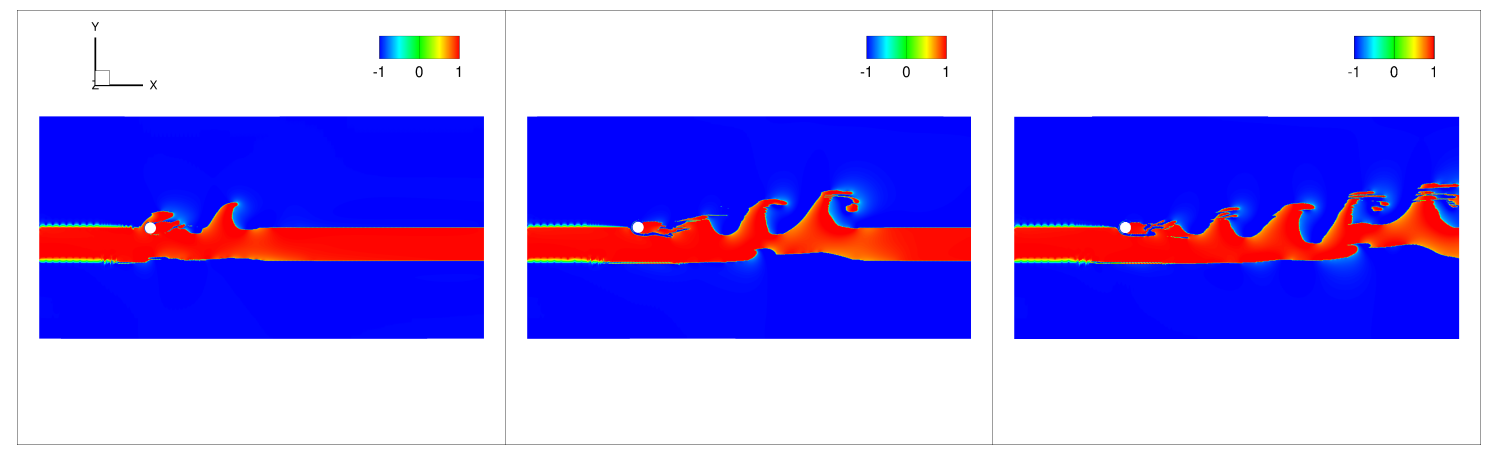

(a) $\mathrm{t}=10.5$

(b) $\mathrm{t}=21$

(c) $\mathrm{t}=31.5$

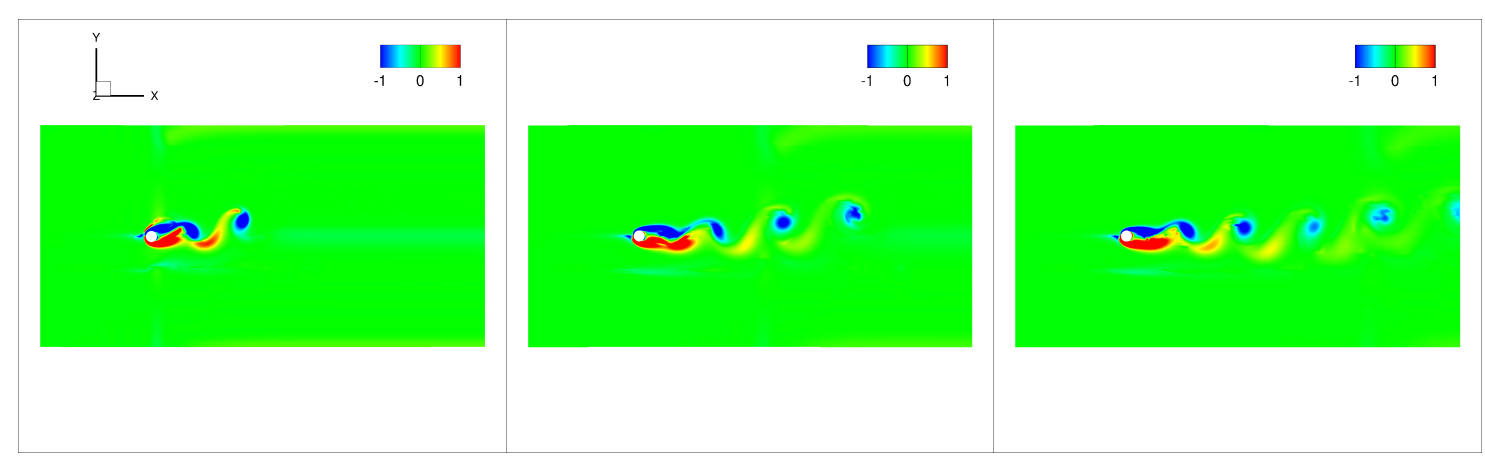

(d) $\mathrm{t}=10.5$

(e) $\mathrm{t}=21$

(f) $\mathrm{t}=31.5$

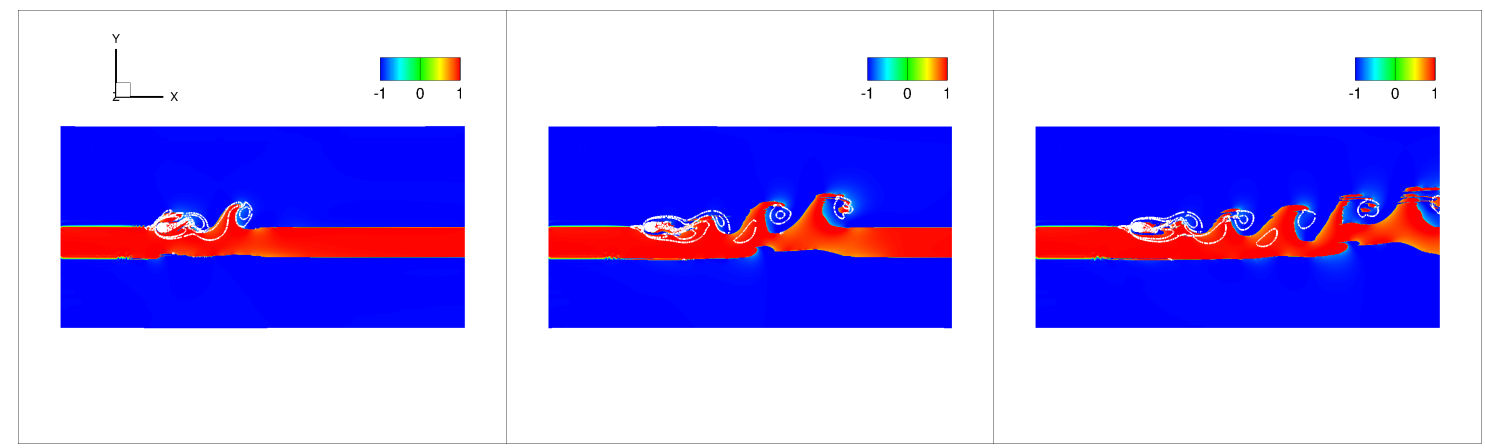

(g) $\mathrm{t}=10.5$

(h) $\mathrm{t}=21$

(i) $\mathrm{t}=31.5$

Figure 12: Free cylinder with $\left(R e, f_{n}\right)=(100,0.167)$. Contour plot at $\mathrm{t}=10.5,21,31.5 .1^{s t}$ row: phasefield; $2^{\text {nd }}$ row: z-vorticity. $3^{\text {rd }}$ row: z-vorticity super-imposed on phase-field contour plot with scaling [-1 $1]$. 


\section{Summary}

A phase-field method for simulating FSI in two-phase flow in the ALE framework is presented. The three-dimensional benchmark test of two-phase co-annular laminar flow in a stationary pipe demonstrates the accuracy of the new multi-physics method in dealing with two-phase flow problems. Furthermore, by comparing results of simulating a flexible pipe conveying single-phase flow from our method with those from a previously validated code [1] we show that our method is physically accurate and efficient for FSI problems. The simulations of pipe conveying two-phase flow and two-phase external cross flow past a circular cylinder demonstrate that our method can capture the complex dynamics of FSI in two-phase flow, including a Kelvin-Helmholtz instability due to density stratification.

In ongoing work we consider additional enhancements of the method. First, to improve the accuracy and generality of the structure solver, we consider using a spectral element method for spatial discretization instead of the Galerkin method and BDF for time discretization instead of the Newmark- $\beta$ scheme. Second, we are replacing the current linear beam model by a nonlinear beam model to obtain more information about very large structure motions and deformations. The structure model can also be replaced by a nonlinear three-dimensional spectral element solver developed recently in [46]. Finally, a more drastic approach is to represent the solid also as a third phase and eliminate totally the ALE formulation in favor of a phase-field representation for the fluid-fluid as well as the fluid-structure interactions. We have followed the work of [41] for the Allen-Cahn equation and the results look promising but the governing equations may not be consistent with the standard elasticity equations. This is a fundamental open issue that we plan to address in the future 
work.

\section{Acknowledgement}

We gratefully acknowledge the support from Chevron-MIT University Partner-ship Program and Sea Grant program in Massachusetts Institute of Technology. High Performance Computing resources were provided by the Center for Computation and Visualization at Brown University, the Argonne Leadership Computing Facility, which is a DOE Office of Science User Facility supported under Contract DE-AC02-06CH11357 and the Extreme Science and Engineering Discovery Environment (XSEDE), which is supported by National Science Foundation grant number ACI-1053575 [48]. 


\section{Appendices}

Appendix A. Boundary and initial conditions for phase-field algorithm in the ALE framework

For the Navier-Stokes equation, i.e., Eq. (1a): We assume periodic boundaries in the z direction and Dirichlet boundary condition on the pipe walls (interface $\sum(t)$ ), which is updated during every iteration by passing the velocity of the structure to the fluid on the interfalece $(\mathbf{u}=\dot{\boldsymbol{\eta}})$.

For the Cahn-Hilliard equation, i.e., Eq. (1c): We assume dynamic contact angle condition for the phase-field variable $\phi(\mathbf{x}, \mathrm{t})$, i.e.,

$$
\begin{array}{r}
\left.n \cdot \nabla \phi\right|_{\Gamma}=0 \\
\left.n \cdot \nabla\left(\nabla^{2} \phi\right)\right|_{\Gamma}=0,
\end{array}
$$

where $\Gamma$ refers to boundaries of the computational domain.

For the mesh velocity, i.e., Eq. (1d)-(1e): We assume vanishing boundary condition for the mesh velocity except on the pipe walls (interface $\sum(t)$ ), which is updated during every iteration by passing the velocity of the fluid to the mesh on the interface $(\mathbf{w}=\dot{\boldsymbol{\eta}})$.

\section{Appendix B. Discretized form for fluid solver - phase-field algorithm in the ALE framework}

This appendix summarizes an algorithm for the incompressible two-phase flow equations (1a)-(1c) in the ALE framework, together with the boundary conditions specified in Appendix A. The main formulation of this algorithm was developed in [22]. However, in the 
ALE framework, we have two more variables to solve, i.e., mesh velocity $\mathbf{w}$ and mesh current configuration $\mathbf{x}$.

We use the projection (splitting) method as in Chapter 8 [49] and also [22]. We use a velocity-correction type strategy to decouple the computation of pressure from that of the velocity, then we split all variable coefficients into a constant (e.g., the average) part and a variable part, and treat the constant part implicitly and the variable part explicitly. For the fourth-order Cahn-Hilliard equation, we decompose it into two independent second-order equations. For the convection (nonlinear) term in the modified NS equation, we use the collocation method. For the resulted pressure and velocity equation from the projection method (Poisson equation for pressure and Helmholtz equation for velocity), we use the Galerkin method.

We re-write (1a) into an equivalent but slightly different form,

$$
\rho\left(\frac{\partial \mathbf{u}}{\partial t}+(\mathbf{u}-\mathbf{w}) \cdot \nabla \mathbf{u}\right)=-\nabla P+\mu \nabla^{2} \mathbf{u}+\nabla \mu \cdot\left(\nabla \mathbf{u}+\nabla \mathbf{u}^{T}\right)-\lambda\left(\nabla^{2} \phi\right) \nabla \phi+\mathbf{f}(\mathbf{x}, t)
$$

where $P=p+\frac{\lambda}{2} \nabla \phi \cdot \nabla \phi$ is an effective pressure, and will also be loosely called pressure.

The formulation of the algorithm is summarized below. Given $\left(\mathbf{u}^{n}, \mathbf{w}^{n}, P^{n}, \phi^{n}, \mathbf{x}^{n+1}\right)$, we successively solve for $\mathbf{u}^{n+1}, \mathbf{w}^{n+1}, P^{n+1}, \phi^{n+1}$ and $\mathbf{x}^{n+1}$ as follows:

$\underline{\text { For } \phi^{n+1}}$

$$
\begin{aligned}
& \frac{\gamma_{0} \phi^{n+1}-\hat{\phi}}{\Delta t}+(\mathbf{u}-\mathbf{w})^{*, n+1} \cdot \nabla \phi^{*, n+1} \\
&=-\lambda \gamma_{1} \nabla^{2}\left[\nabla^{2} \phi^{n+1}-\frac{S}{\eta^{2}}\left(\phi^{n+1}-\phi^{*, n+1}\right)-h\left(\phi^{*, n+1}\right)\right]+g^{n+1}
\end{aligned}
$$




$$
\begin{aligned}
& \left.\mathbf{n} \cdot \nabla\left[\nabla^{2} \phi^{n+1}-\frac{S}{\eta^{2}}\left(\phi^{n+1}-\phi^{*, n+1}\right)-h\left(\phi^{*, n+1}\right)\right]\right|_{\partial \Omega}=g_{a}^{n+1}, \\
& \left.\mathbf{n} \cdot \nabla \phi^{n+1}\right|_{\partial \Omega}=-\frac{1}{\lambda} f_{w}^{\prime}\left(\phi^{*, n+1}\right)+g_{b}^{n+1} .
\end{aligned}
$$

For $P^{n+1}$

$$
\begin{gathered}
\frac{\gamma_{0} \tilde{\mathbf{u}}^{n+1}-\hat{\mathbf{u}}}{\Delta t}+\frac{1}{\rho_{0}} \nabla P^{n+1}=-\mathbf{N}\left(\mathbf{u}^{n}\right)+\left(\frac{1}{\rho_{0}}-\frac{1}{\rho^{n+1}}\right) \nabla P^{n}-\frac{\mu^{n+1}}{\rho^{n+1}} \nabla \times \boldsymbol{\omega}^{n} \\
+\frac{1}{\rho^{n+1}} \nabla \mu^{n+1} \cdot \mathbf{D}\left(\mathbf{u}^{n}\right)-\frac{\lambda}{\rho^{n+1}} \nabla^{2} \phi^{n+1} \nabla \phi^{n+1}+\frac{1}{\rho^{n+1}} \mathbf{f}^{n+1}, \\
\nabla \cdot \tilde{\mathbf{u}}^{n+1}=0, \\
\left.\mathbf{n} \cdot \tilde{\mathbf{u}}^{n+1}\right|_{\partial \Omega}=\mathbf{n} \cdot \mathbf{u}_{\mathbf{b}}{ }^{n+1} .
\end{gathered}
$$

For $\mathbf{u}^{n+1}$

$$
\begin{gathered}
\frac{\gamma_{0} \mathbf{u}^{n+1}-\gamma_{0} \tilde{\mathbf{u}}^{n+1}}{\Delta t}-\nu_{m} \nabla^{2} \mathbf{u}^{n+1}=-\mathbf{N}\left(\mathbf{u}^{*, n+1}\right)+\mathbf{N}\left(\mathbf{u}^{n}\right)+\nu_{m} \nabla \times \boldsymbol{\omega}^{*, n+1} \\
+\left(\frac{1}{\rho_{0}}-\frac{1}{\rho^{n+1}}\right) \nabla\left(P^{n+1}-P^{n}\right)-\frac{\mu^{n+1}}{\rho^{n+1}} \nabla \times\left(\boldsymbol{\omega}^{*, n+1}-\boldsymbol{\omega}^{n}\right) \\
+\frac{1}{\rho^{n+1}} \nabla \mu^{n+1} \cdot\left[\mathbf{D}\left(\mathbf{u}^{*, n+1}\right)-\mathbf{D}\left(\mathbf{u}^{n}\right)\right] \\
\left.\mathbf{u}^{n+1}\right|_{\partial \Omega}=\mathbf{u}_{\mathbf{b}}{ }^{n+1} .
\end{gathered}
$$

For $\mathbf{w}^{n+1}$

$$
\nabla^{2} \mathbf{w}=0
$$




$$
\mathbf{w}=\frac{\partial \eta}{\partial t}, \text { on } \Sigma(t)
$$

For $\mathbf{x}^{n+1}$

$$
\frac{\mathbf{x}^{n}-\sum_{i=1}^{J} \hat{\alpha}_{i} \mathbf{x}^{n-i}}{\Delta t}=\sum_{i=1}^{J} \hat{\hat{\alpha}}_{i} \mathbf{w}^{n-i} .
$$

In the above equations, $\tilde{\mathbf{u}}^{n+1}$ is an intermediate velocity, an approximation of $\mathbf{u}^{n+1} \cdot \mathbf{N}(\mathbf{u})=$ $(\mathbf{u}-\mathbf{w}) \cdot \nabla \mathbf{u}$, and $\mathbf{D}(\mathbf{u})=\nabla \mathbf{u}+\nabla \mathbf{u}^{T} . f_{w}(\phi)$ is the fluid-solid interfacial tension function defined on the wall, with $f_{w}(\phi)=\sigma \cos \left(\theta_{s}\right) \frac{\phi\left(\phi^{2}-3\right)}{4}+\frac{1}{2}\left(\sigma_{w 1}+\sigma_{w 2}\right)$. Here $\sigma_{w 1}$ and $\sigma_{w 2}$ is the fluid one and fluid two solid interfacial tension. When the contact angle is $90, f_{w}(\phi)=0$. Variables and parameters are defined as in $[22,23]$ and we summarize them as follows. If $\chi$ denotes a generic variable, $\hat{\chi}$ and $\chi^{*, n+1}$ denote the extrapolation from previous time steps. $\rho^{n+1}$ and $\mu^{n+1}$ are respectively the density and dynamic viscosity at time step $(n+1)$, determined from the equations below :

$$
\begin{array}{r}
\rho(\phi)=\frac{1}{2}\left(\rho_{1}+\rho_{2}\right)+\frac{1}{2}\left(\rho_{1}-\rho_{2}\right) \phi \\
\mu(\phi)=\frac{1}{2}\left(\mu_{1}+\mu_{2}\right)+\frac{1}{2}\left(\mu_{1}-\mu_{2}\right) \phi .
\end{array}
$$

The constant $\rho_{0}$ is given by $\rho_{0}=\min \left(\rho_{1}, \rho_{2}\right)$. The parameter $\nu_{m}$ is a chosen constant satisfying $\nu_{m} \geqslant \frac{1}{2}\left(\frac{\mu_{1}}{\rho_{1}}+\frac{\mu_{2}}{\rho_{2}}\right) . S$ is a chosen constant satisfying condition $S \geqslant \eta^{2} \sqrt{\frac{4 \gamma_{0}}{\lambda \gamma_{1} \Delta t}}$. Note here $\mathbf{u}_{\mathbf{b}}$ is the prescribed velocity on the domain boundary $\Omega, g_{a}$ and $g_{b}$ are prescribed scalar field functions on domain boundary $\Omega, f_{w}(\phi)$ is the fluid-solid interfacial tension function defined on the wall and $h(\phi)$ is given by $h(\phi)=\frac{1}{\eta^{2}} \phi\left(\phi^{2}-1\right)$.

To implement the above scheme, we reformulate (10a) into two de-coupled Helmholtz 
type equations (see [22] for details):

$$
\begin{aligned}
& \nabla^{2} \psi^{n+1}-\left(\alpha+\frac{S}{\eta^{2}}\right) \psi^{n+1}=Q, \\
& \nabla^{2} \phi^{n+1}+\alpha \phi^{n+1}=\psi^{n+1}
\end{aligned}
$$

where $\psi^{n+1}$ is an auxiliary variable,

$$
Q=\frac{1}{\lambda \gamma_{1}}\left(g^{n+1}-(\mathbf{u}-\mathbf{w})^{*, n+1} \cdot \nabla \phi^{*, n+1}+\frac{\hat{\phi}}{\Delta t}\right)+\nabla^{2}\left[h\left(\phi^{*, n+1}\right)-\frac{S}{\eta^{2}} \phi^{*, n+1}\right],
$$

and $\alpha$ is a constant given by $\alpha=-\frac{S}{2 \eta^{2}}\left(1-\sqrt{1-\frac{4 \gamma_{0}}{\lambda \gamma_{1} \Delta t} \frac{\eta^{4}}{S^{2}}}\right)$. In light of (18) and (10c), the boundary condition (10b) can be transformed to

$\left.\mathbf{n} \cdot \nabla \psi^{n+1}\right|_{\partial \Omega}=\left.\left(\alpha+\frac{S}{\eta^{2}}\right)\left(-\frac{1}{\lambda} f_{w}^{\prime}\left(\phi^{*, n+1}\right)+g_{b}^{n+1}\right)\right|_{\partial \Omega}+\left.\mathbf{n} \cdot \nabla\left[h\left(\phi^{*, n+1}\right)-\frac{S}{\eta^{2}} \phi^{*, n+1}\right]\right|_{\partial \Omega}+g_{a}^{n+1}$

Let $\varphi \in H^{1}(\Omega)$ denote the test function. By taking the $L^{2}$ inner product between equation (17) and $\varphi$, we obtain the weak form for $\psi$,

$$
\begin{aligned}
& \int_{\Omega} \nabla \psi^{n+1} \cdot \nabla \varphi+\left(\alpha+\frac{S}{\eta^{2}}\right) \int_{\Omega} \psi^{n+1} \varphi \\
&=-\int_{\Omega} \frac{1}{\lambda \gamma_{1}}\left[g^{n+1}-(\mathbf{u}-\mathbf{w})^{*, n+1} \cdot \nabla \phi^{*, n+1}+\frac{\hat{\phi}}{\Delta t}\right] \varphi+\int_{\Omega} \nabla\left[h\left(\phi^{*, n+1}\right)-\frac{S}{\eta^{2}} \phi^{*, n+1}\right] \cdot \nabla \varphi+\int_{\partial \Omega} g_{a}^{n+1} \varphi \\
& \quad+\left(\alpha+\frac{S}{\eta^{2}}\right) \int_{\partial \Omega}\left(-\frac{1}{\lambda} f_{w}^{\prime}\left(\phi^{*, n+1}\right)+g_{b}^{n+1}\right) \varphi, \quad \forall \varphi \in H^{1}(\Omega), \quad(21)
\end{aligned}
$$

where we have used (20). Similarly, one can obtain from (18) the weak form for $\phi^{n+1}$,

$$
\int_{\Omega} \nabla \phi^{n+1} \cdot \nabla \varphi-\alpha \int_{\Omega} \phi^{n+1} \varphi=-\int_{\Omega} \psi^{n+1} \varphi+\int_{\partial \Omega}\left(-\frac{1}{\lambda} f_{w}^{\prime}\left(\phi^{*, n+1}\right)+g_{b}^{n+1}\right) \varphi, \quad \forall \varphi \in H^{1}(\Omega) .
$$

Equations (27) and (28) can be readily discretized using $C^{0}$ spectral elements or finite elements. They are successively solved for $\psi^{n+1}$ and $\phi^{n+1}$ in an un-coupled fashion. 
Let $q \in H^{1}(\Omega)$ denote the test function. Take the $L^{2}$-inner product between (11a) and $\nabla q$, and we obtain the following weak form for $P^{n+1}$,

$$
\begin{aligned}
\int_{\Omega} \nabla P^{n+1} \cdot \nabla q= & \rho_{0} \int_{\Omega}\left[\mathbf{G}+\nabla\left(\frac{\mu^{n+1}}{\rho^{n+1}}\right) \times \boldsymbol{\omega}^{\boldsymbol{n}}\right] \cdot \nabla q-\rho_{0} \int_{\partial \Omega} \frac{\mu^{n+1}}{\rho^{n+1}} \mathbf{n} \times \boldsymbol{\omega}^{n} \cdot \nabla q \\
& -\frac{\gamma_{0} \rho_{0}}{\Delta t} \int_{\partial \Omega} \mathbf{n} \cdot \mathbf{u}_{\mathbf{b}}^{n+1} q, \quad \forall q \in H^{1}(\Omega),
\end{aligned}
$$

where

$\mathbf{G}=\frac{1}{\rho^{n+1}}\left[\mathbf{f}^{n+1}-\lambda\left(\psi^{n+1}-\alpha \phi^{n+1}\right) \nabla \phi^{n+1}+\nabla \mu^{n+1} \cdot \mathbf{D}\left(\mathbf{u}^{n}\right)\right]+\frac{\hat{\mathbf{u}}}{\Delta t}-\mathbf{N}\left(\mathbf{u}^{n}\right)+\left(\frac{1}{\rho_{0}}-\frac{1}{\rho^{n+1}}\right) \nabla P^{n}$,

and $\boldsymbol{\omega}=\nabla \times \mathbf{u}$ denotes the vorticity, and we have used equations (11b) and (11c).

Let $H_{0}^{1}(\Omega)=\left\{v \in H^{1}(\Omega):\left.v\right|_{\partial \Omega}=0\right\}$, and $\varphi \in H_{0}^{1}(\Omega)$ denote the test function. Take the $L^{2}$-inner product between $(12 \mathrm{a})$ and $\varphi$, and note that the intermediate velocity can be substituted by, according to (11a), $\frac{\gamma_{0}}{\Delta t} \tilde{\mathbf{u}}^{n+1}=\mathbf{G}-\frac{\mu^{n+1}}{\rho^{n+1}} \nabla \times \boldsymbol{\omega}^{n}-\frac{1}{\rho_{0}} \nabla P^{n+1}$. We then obtain the weak form about $\mathbf{u}^{n+1}$ :

$$
\begin{aligned}
\frac{\gamma_{0}}{\nu_{m} \Delta t} \int_{\Omega} \varphi \mathbf{u}^{n+1}+\int_{\Omega} \nabla \varphi \cdot \nabla \mathbf{u}^{n+1} & =\frac{1}{\nu_{m}} \int_{\Omega}\left[\mathbf{R}+\nabla\left(\frac{\mu^{n+1}}{\rho^{n+1}}\right) \times \boldsymbol{\omega}^{*, n+1}\right] \varphi \\
& -\frac{1}{\nu_{m}} \int_{\Omega}\left(\frac{\mu^{n+1}}{\rho^{n+1}}-\nu_{m}\right) \boldsymbol{\omega}^{*, n+1} \times \nabla \varphi \\
& -\frac{1}{\nu_{m}} \int_{\partial \Omega}\left(\frac{\mu^{n+1}}{\rho^{n+1}}-\nu_{m}\right) \mathbf{n} \times \boldsymbol{\omega}^{*, n+1} \varphi, \quad \forall \varphi \in H_{0}^{1}(\Omega) .
\end{aligned}
$$

where

$$
\begin{aligned}
\mathbf{R}=\frac{1}{\rho^{n+1}}\left[\mathbf{f}^{n+1}-\lambda\left(\psi^{n+1}-\alpha \phi^{n+1}\right) \nabla \phi^{n+1}+\nabla \mu^{n+1}\right. & \left.\mathbf{D}\left(\mathbf{u}^{*, n+1}\right)\right] \\
& +\frac{\hat{\mathbf{u}}}{\Delta t}-\mathbf{N}\left(\mathbf{u}^{*, n+1}\right)-\frac{1}{\rho^{n+1}} \nabla P^{n+1} .
\end{aligned}
$$

The weak forms (29) and (31) can be readily discretized in space with $C^{0}$ spectral elements or finite elements. 
We now consider the spatial discretization of (10)-(12), following the similar way in [22]. Let $X_{h}$ denote domain $\mathrm{X}$ discretized with a spectral element mesh, and $C_{h}$ denote the boundary of $X_{h}$. Let $X_{h} \subset H^{1}\left(\Omega_{h}\right)^{d}$ and $M_{h} \subset H^{1}\left(\Omega_{h}\right)$ respectively denote the approximation spaces of the phase-field variable, $\boldsymbol{\phi}_{\boldsymbol{h}}^{k+1}$, velocity, $\mathbf{u}_{\mathbf{h}}{ }^{k+1}$ and pressure, $\mathbf{p}_{\mathbf{h}}{ }^{k+1}$. Then the fully discretized version of the system (10)-(12) is: Find $\boldsymbol{\phi}_{\boldsymbol{h}}^{k+1} \in H^{1}\left(\Omega_{h}\right), \mathbf{u}_{\mathbf{h}}{ }^{k+1} \in H^{1}\left(\Omega_{h}\right)^{d}$ and $\mathbf{p}_{\mathbf{h}}{ }^{k+1} \in H^{1}\left(\Omega_{h}\right)$ such that,

$$
\begin{gathered}
\int_{\Omega_{h}} \nabla \psi_{h}^{n+1} \cdot \nabla \varphi_{h}+\left(\alpha+\frac{S}{\eta^{2}}\right) \int_{\Omega_{h}} \psi_{h}^{n+1} \varphi_{h} \\
=-\int_{\Omega_{h}} \frac{1}{\lambda \gamma_{1}}\left[g_{h}^{n+1}-\left(\mathbf{u}_{\mathbf{h}}-\mathbf{w}_{\mathbf{h}}\right)^{*, n+1} \cdot \nabla \phi_{h}^{*, n+1}+\frac{\hat{\phi_{h}}}{\Delta t}\right] \varphi_{h}+\int_{\Omega_{h}} \nabla\left[h\left(\phi_{h}^{*, n+1}\right)-\frac{S}{\eta^{2}} \phi_{h}^{*, n+1}\right] \cdot \nabla \varphi_{h}+\int_{\partial \Omega_{h}} g_{a_{h}}^{n+1} \\
\quad+\left(\alpha+\frac{S}{\eta^{2}}\right) \int_{\partial \Omega_{h}}\left(-\frac{1}{\lambda} f_{w}{ }^{\prime}\left(\phi_{h}^{*, n+1}\right)+g_{b h}^{n+1}\right) \varphi_{h}, \quad \forall \varphi_{h} \in H^{1}\left(\Omega_{h}\right) . \quad(27)
\end{gathered}
$$

Similarly, one can obtain from (22) the weak form for $\phi_{h}^{n+1}$,

$$
\int_{\Omega_{h}} \nabla \phi_{h}^{n+1} \cdot \nabla \varphi_{h}-\alpha \int_{\Omega_{h}} \phi_{h}^{n+1} \varphi_{h}=-\int_{\Omega_{h}} \psi_{h}^{n+1} \varphi_{h}+\int_{\partial \Omega_{h}}\left(-\frac{1}{\lambda} f_{w}{ }^{\prime}\left(\phi_{h}^{*, n+1}\right)+g_{b h}^{n+1}\right) \varphi_{h}, \quad \forall \varphi_{h} \in H^{1}\left(\Omega_{h}\right) .
$$

They are successively solved for $\psi_{h}^{n+1}$ and $\phi_{h}^{n+1}$ in an un-coupled fashion.

Let $q_{h} \in H^{1}\left(\Omega_{h}\right)$ denote the test function. Take the $L^{2}$-inner product between $11(\mathrm{a})-11(\mathrm{c})$ and $\nabla q_{h}$, and we obtain the following weak form for $P_{h}^{n+1}$,

$$
\begin{aligned}
\int_{\Omega_{h}} \nabla P_{h}^{n+1} \cdot \nabla q_{h}= & \rho_{0} \int_{\Omega_{h}}\left[\mathbf{G}+\nabla\left(\frac{\mu^{n+1}}{\rho^{n+1}}\right) \times \boldsymbol{\omega}_{\boldsymbol{h}}^{n}\right] \cdot \nabla q_{h}-\rho_{0} \int_{\partial \Omega_{h}} \frac{\mu^{n+1}}{\rho^{n+1}} \mathbf{n} \times \boldsymbol{\omega}_{\boldsymbol{h}}^{n} \cdot \nabla q_{h} \\
& -\frac{\gamma_{0} \rho_{0}}{\Delta t} \int_{\partial \Omega_{h}} \mathbf{n} \cdot \mathbf{u}_{\mathbf{b} h}^{n+1} q_{h}, \quad \forall q_{h} \in H_{h}^{1}(\Omega),
\end{aligned}
$$

where

$$
\mathbf{G}_{\mathbf{h}}=\frac{1}{\rho^{n+1}}\left[\mathbf{f}_{\mathbf{h}}{ }^{n+1}-\lambda\left(\psi_{h}^{n+1}-\alpha \phi_{h}^{n+1}\right) \nabla \phi_{h}^{n+1}+\nabla \mu^{n+1} \cdot \mathbf{D}\left(\mathbf{u}_{\mathbf{h}}{ }^{n}\right)\right]+\frac{\hat{\mathbf{u}_{\mathbf{h}}}}{\Delta t}-\mathbf{N}\left(\mathbf{u}_{\mathbf{h}}{ }^{n}\right)+\left(\frac{1}{\rho_{0}}-\frac{1}{\rho^{n+1}}\right) \nabla P_{h}^{n},
$$


and $\boldsymbol{\omega}_{\boldsymbol{h}}=\nabla \times \mathbf{u}_{\mathbf{h}}$ denotes the vorticity.

Let $H_{0}^{1}\left(\Omega_{h}\right)=\left\{v \in H^{1}\left(\Omega_{h}\right):\left.v\right|_{\partial \Omega_{h}}=0\right\}$, and $\varphi_{h} \in H_{0}^{1}\left(\Omega_{h}\right)$ denote the test function. Take the $L^{2}$-inner product between $(23)$ and $\varphi_{h}$, and note that the intermediate velocity can be substituted by, according to Eqns. 11(a)-11(c), $\frac{\gamma_{0}}{\Delta t} \tilde{\mathbf{u}}_{\mathbf{h}}{ }^{n+1}=\mathbf{G}-\frac{\mu^{n+1}}{\rho^{n+1}} \nabla \times \boldsymbol{\omega}_{\boldsymbol{h}}^{n}-\frac{1}{\rho_{0}} \nabla P_{h}^{n+1}$. We then obtain the weak form about $\mathbf{u}_{\mathbf{h}}{ }^{n+1}$ :

$$
\begin{aligned}
\frac{\gamma_{0}}{\nu \Delta t} \int_{\Omega_{h}} \varphi_{h} \mathbf{u}_{\mathbf{h}}{ }^{n+1}+\int_{\Omega_{h}} \nabla \varphi_{h} \cdot \nabla \mathbf{u}_{\mathbf{h}}{ }^{n+1} & =\frac{1}{\nu_{m}} \int_{\Omega_{h}}\left[\mathbf{R}+\nabla\left(\frac{\mu^{n+1}}{\rho^{n+1}}\right) \times \boldsymbol{\omega}_{\boldsymbol{h}}^{*, n+1}\right] \varphi_{h} \\
& -\frac{1}{\nu_{h m}} \int_{\Omega_{h}}\left(\frac{\mu^{n+1}}{\rho^{n+1}}-\nu_{m}\right) \boldsymbol{\omega}_{\boldsymbol{h}}^{*, n+1} \times \nabla \varphi_{h} \\
& -\frac{1}{\nu_{m}} \int_{\partial \Omega_{h}}\left(\frac{\mu^{n+1}}{\rho^{n+1}}-\nu_{m}\right) \mathbf{n} \times \boldsymbol{\omega}_{\boldsymbol{h}}^{*, n+1} \varphi_{h}, \quad \forall \varphi \in H_{0}^{1}\left(\Omega_{h}\right) .
\end{aligned}
$$

where

$$
\begin{aligned}
\mathbf{R}_{\mathbf{h}}=\frac{1}{\rho^{n+1}}\left[\mathbf{f}_{\mathbf{h}}{ }^{n+1}-\lambda\left(\psi_{h}^{n+1}-\alpha \phi_{h}^{n+1}\right) \nabla \phi_{h}^{n+1}+\right. & \left.\nabla \mu^{n+1} \cdot \mathbf{D}\left(\mathbf{u}_{\mathbf{h}}{ }^{*, n+1}\right)\right] \\
& +\frac{\hat{\mathbf{u}_{\mathbf{h}}}}{\Delta t}-\mathbf{N}\left(\mathbf{u}_{\mathbf{h}}{ }^{*, n+1}\right)-\frac{1}{\rho^{n+1}} \nabla P_{h}^{n+1} .
\end{aligned}
$$

The final algorithm consists of: (i) solving (27) and (28) successively for $\psi^{n+1}$ and $\phi^{n+1}$, (ii) solving (29) for $P^{n+1}$, (iii) solving (31) for $\mathbf{u}^{n+1}$, (iv) solving (13a) and (13b) for $\mathbf{w}^{n+1}$, and (v) solving (14a) for $\mathbf{x}^{n+1}$.

Note here the mesh is updated every time step and the coefficient matrices are recomputed every time step.

Finally, [22] discussed the modification of equation (28) when computing $\rho^{n+1}$ and $\mu^{n+1}$ when the density ratio of the two fluids becomes very large or conversely very small (typically beyond $10^{2}$ or below $10^{-2}$ ). 


\section{Appendix C. Coefficient matrices for structure equation}

The coefficients of the tensor form of the beam equation, i.e., Eq. (2), are given here:

$$
\begin{aligned}
& M_{i j}^{u}=\int_{0}^{L} \phi_{i}^{u}(x) \phi_{j}^{u}(x) d x \quad K_{i j}^{u}=E I \int_{0}^{L}\left(\phi_{i}^{u}\right)^{\prime \prime}(x)\left(\phi_{j}^{u}\right)^{\prime \prime}(x) d x+T \int_{0}^{L}\left(\phi_{i}^{u}\right)^{\prime}(x)\left(\phi_{j}^{u}\right)^{\prime}(x) d x \\
& Q_{\{x, y, z\}}=\int_{0}^{1}\left(F_{\{x, y, z\}}, \phi_{j}\right)
\end{aligned}
$$

\section{Appendix D. Non-dimensionalization of variables and governing equations}

Let the superscript in $(.)^{*}$ denote the dimensional variable and (.) corresponding to nondimensional variable. First we show how we non-dimensionalize the variables for the fluid solver in Section 3. Note $\alpha_{1,2}$ denotes the volume fraction of the first and second fluid. $U_{0}$ and $L$ denote the characteristic velocity and length.

$$
\left\{\begin{array}{l}
x=\frac{x^{*}}{L}, t=\frac{t^{*}}{L / U_{0}}, \\
p=\frac{p^{*}}{\rho_{1} U_{0}^{2}}, u=\frac{u^{*}}{U_{0}} \\
\eta=C_{n}=\frac{\eta^{*}}{L} \text { (Cahn number), } \sigma=\frac{1}{W_{e}}=\frac{\sigma^{*}}{\rho_{1} U_{0}^{2} L}, \lambda=\frac{\lambda^{*}}{\rho_{1} U_{0}{ }^{2} L}=\frac{3}{2 \sqrt{2}} \sigma^{*} \eta^{*} \\
\gamma_{1}=\frac{\gamma_{1}^{*}}{\frac{L}{\left(\rho_{1} U_{0}\right)}}=\frac{W_{e} C_{n}}{P_{e}}, \\
\rho(\phi)=\frac{\rho^{*}(\phi)}{\rho_{1}}=\frac{\rho_{1} \alpha_{1}+\rho_{2} \alpha_{2}}{\rho_{1}}
\end{array}\right.
$$

where $W_{e}=\frac{\rho_{1} U_{0}^{2} L}{\sigma^{*}}$ (Weber number) and $P_{e}=\frac{U_{0} \eta^{*} L}{\sigma^{*} \gamma_{1}^{*}}$ (Peclet number). 
Correspondingly, the nondimensional governing equations are given by

$$
\begin{aligned}
& \rho\left(\frac{\partial \mathbf{u}}{\partial t}+(\mathbf{u}-\mathbf{w}) \cdot \nabla \mathbf{u}\right)=-\nabla p+\nabla \cdot\left[\mu\left(\nabla \mathbf{u}+\nabla \mathbf{u}^{T}\right)\right]-\lambda \nabla \cdot(\nabla \phi \nabla \phi)+\mathbf{f}(\mathbf{x}, t) \\
& \nabla \cdot \mathbf{u}=0 \\
& \frac{\partial \phi}{\partial t}+(\mathbf{u}-\mathbf{w}) \cdot \nabla \phi=-\lambda \gamma_{1} \nabla^{2}\left[\nabla^{2} \phi-h(\phi)\right]+g(\mathbf{x}, t), \\
& \nabla^{2} \mathbf{w}=0, \text { on } \Omega_{f} \\
& \mathbf{w}=\frac{\partial \eta}{\partial t}, \text { on } \Sigma(t)
\end{aligned}
$$

where $h(\phi)=\frac{1}{\eta^{2}} \phi\left(\phi^{2}-1\right)$, and $f=\frac{L}{\rho_{1} U_{0}^{2}} f, g=\frac{L}{U_{0}} g$. The boundary conditions for the velocity and the phase-field in Appendix A can be non-dimensionalized using the dimensionless parameters in Eq. 34.

For the structure solver, we use dimensionless parameter $T=T^{*} L^{2} / E I$ and $\eta\left(q_{x}, q_{y}, q_{z}\right)^{*}=$ $\eta\left(q_{x}, q_{y}, q_{z}\right) / L$

The system therefore involves several non-dimensional parameters: density ratio $\frac{\rho_{2}}{\rho_{1}}$, dynamic viscosity ratio $\frac{\mu_{2}}{\mu_{1}}$, Cahn number $C_{n}$, Weber number $W_{e}$, Peclet number $P_{e}$, Reynolds number $R e$, and contact angle $\theta_{s}$. When the gravity is taken into account, it also involves the Froude number $F_{r}=\frac{U_{0}}{\sqrt{g_{r} L}}$, where $g_{r}$ is the gravitational acceleration. We note that, when the flow variables and physical parameters are non-dimensionalized as given above, the nondimensional governing equations and the boundary conditions have the same forms as the original dimensional ones. Therefore, we drop the superscript $(*)$ and understand that the variables and equations have been appropriately normalized.

Here for the tension related parameter $I_{p}$, which indicates the instability for pipe conveying fluids, defined as $I_{p}=\frac{m_{f} U_{0}^{2}(e-0.125)}{T(e+1)} . L_{2}(\mathbf{u}(u, v, w))$, a measure of the velocity, is defined as $L_{2}(\mathbf{u}(u, v, w))=\int_{\Omega}(u, v, w)^{2} /($ volume of the domain). Re for two-phase flow is defined as 
$R e=\overline{\rho_{f}} U_{0} L / \overline{\mu_{f}}$, where $\overline{\rho_{f}}=\rho_{1} \alpha_{1}+\rho_{2} \alpha_{2}$ and $\overline{\mu_{f}}=\mu_{1} \alpha_{1}+\mu_{2} \alpha_{2}$. Here we use weighted density $\overline{\rho_{f}}$ and viscosity $\overline{\mu_{f}}$ for calculating $R e$, which is different from space and time dependent density $\rho(\phi(x ; t))$ and viscosity $\mu(\phi(x ; t))$ as we used in the algorithm and fluid force calculation.

\section{Appendix E. Boundary and initial conditions for two-phase co-annular laminar flow in a stationary pipe}

Below are the initial and boundary conditions for two-phase co-annular laminar flow in a stationary pipe. For the velocity, we use the following fully developed velocity profile at the inlet and also as the initial set up. It can be found in [50]:

$$
\left\{\begin{array}{l}
U_{1}(R)=\frac{u_{1}(r)}{\bar{u}}=\frac{C\left[1-\delta_{1}^{2}+\hat{\mu}\left(\delta_{1}^{2}-R^{2}\right)\right]}{\delta_{1}^{n+3}\left(\hat{\left.\mu_{1}+1\right)}\right.}, 0 \leq R \leq \delta_{1} \\
U_{2}(R)=\frac{u_{2}(r)}{\bar{u}}=\frac{C\left[1-R^{2}\right]}{\delta_{1}^{n+3}\left(\hat{\left.\mu_{1}+1\right)}\right.}, \delta_{1} \leq R \leq 1
\end{array}\right.
$$

where $R=\frac{r}{r_{2}}, \hat{u}=\frac{\mu_{2}}{\mu_{1}}, \delta_{1}=\frac{r_{1}}{r_{2}}, C=2$. For the outlet, we have the $\partial \mathbf{u} / \partial n=0$ condition imposed. For the phase-field, the profile at the inlet is $\phi=-\tanh \left(\left(\sqrt{\left(x^{2}+y^{2}\right)}-R 1\right) / \delta\right)$ with $\mathrm{R} 1=0.4$ and $\delta=\sqrt{2} \eta$, which is also the initial profile setup. Contact-angle boundary conditions are imposed on the pipe walls and outlet with $\theta_{s}=90$.

\section{Appendix F. Boundary and initial conditions for pipe conveying single- and two- phase flow}

Below are the initial and boundary conditions for pipe conveying single-phase flow. For the velocity, we use periodic boundry conditions in the z direction. No-slip boundary are imposed on pipe walls. For the phase-field, contact-angle boundary conditions are imposed on the pipe walls and outlet with $\theta_{s}=90$. 
Below are the initial and boundary conditions for pipe conveying two-phase flow. For the velocity, conditions are the same as the single-phase flow case. For the phase-field, the profile at the inlet is $\phi=-\tanh \left(\left(\sqrt{\left(x^{2}+y^{2}\right)}-R 1\right) / \delta\right)$ with $\mathrm{R} 1=0.3$ and $\delta=\sqrt{2} \eta$, which is also the initial profile setup. For the phase-field variable $\phi(\mathbf{x}, t)$, we use interface thickness $\eta=0.001$ and surface tension 0.001 .

\section{Appendix G. Boundary and initial conditions for two-phase external cross flow past a circular cylinder}

Below are the initial and boundary conditions for two-phase external cross flow past a circular cylinder. For the velocity, we use the periodic boundry conditions in the y and $\mathrm{z}$ directions. At the inlet $(\mathrm{x}=-10)$, we use Dirichlet boundary $\mathbf{u}=\mathbf{1}$. At the outlet $(\mathrm{x}=30)$, we have the $\partial \mathbf{u} / \partial n=0$ condition imposed. For the phase-field, the profile at the inlet is $\phi=-\tanh \left(\left(y-Y_{0}\right) / \delta\right) \tanh \left(\left(y-Y_{1}\right) / \delta\right)$ with $Y_{0}=0, Y_{1}=-3$ and $\delta=\sqrt{2} \eta$, which is also the initial profile setup. Contact-angle boundary conditions are imposed on the pipe walls and outlet with $\theta_{s}=90$. For the phase-field variable $\phi(\mathbf{x}, t)$, we use interface thickness $\eta=$ 0.001 and surface tension 0.001 .

\section{References}

[1] D. J. Newman, G. E. Karniadakis, A direct numerical simulation study of flow past a freely vibrating cable, Journal of Fluid Mechanics 344 (1997) 95-136.

[2] O. Baker, et al., Design of pipelines for the simultaneous flow of oil and gas, in: Fall Meeting of the Petroleum Branch of AIME, Society of Petroleum Engineers, 1953. 
[3] M. J. Pettigrew, C. E. Taylor, Two-phase flow-induced vibration: An overview (survey paper), Journal of Pressure Vessel Technology 116 (3) (1994) 233-253.

[4] J. L. Riverin, E. De Langre, M. J. Pettigrew, Fluctuating forces caused by internal twophase flow on bends and tees, Journal of Sound and Vibration 298 (4) (2006) 1088-1098.

[5] S. Vist, J. Pettersen, Two-phase flow distribution in compact heat exchanger manifolds, Experimental Thermal and Fluid Science 28 (2) (2004) 209-215.

[6] F. Mayinger, Classification and applications of two-phase flow heat exchangers, in: TwoPhase Flow Heat Exchangers, Springer, 1988, pp. 3-27.

[7] D. A. Ervine, Air entrainment in hydraulic structures: A review, Proceedings of the ICE-Water Maritime and Energy 130 (3) (1998) 142-153.

[8] F. Yilmaz, M. Y. Gundogdu, A critical review on blood flow in large arteris; relevance to blood rheology, viscosity models, and physiologic conditions, Korea-Australia Rheology Journal 20 (2008) 197-211.

[9] F. Yilmaz, M. Y. Gundogdu, Analysis of conventional drag and lift models for multiphase CFD modeling of blood flow, Korea-Australia Rheology Journal 21 (3) (2009) $161-173$.

[10] R. A. Ibrahim, Overview of mechanics of pipes conveying fluids - Part I: Fundamental studies, Journal of Pressure Vessel Technology 132 (3) (2010) 034001-1-034001-31.

[11] M. P. Paidoussis, Fluid-structure interactions-slender structures and axial flow, volume 1, Academic Press, San Diego, California 92101-4495, USA, 1998. 
[12] T. S. Yih, P. Griffith, Unsteady momentum fluxes in two-phase flow and the vibration of nuclear system components., Tech. rep., Stone and Webster Engineering Corp., Boston, Mass.; Massachusetts Inst. of Tech., Cambridge (1970).

[13] Y. Joo, V. K. Dhir, An experimental study of drag on a single tube and on a tube in an array under two-phase cross flow, International Journal of Multiphase Flow 20 (6) (1994) 1009-1019.

[14] A. Inoue, Y. Kozawa, M. Yokosawa, S. Aoki, Studies on two-phase cross flow. Part I: Flow characteristics around a cylinder, International Journal of Multiphase Flow 12 (2) (1986) 149-167.

[15] M. Yokosawa, Y. Kozawa, A. Inoue, S. Aoki, Studies on two-phase cross flow. Part II: Transition Reynolds number and drag coefficient, International Journal of Multiphase Flow 12 (2) (1986) 169-184.

[16] J. P. Hulin, C. Fierfort, R. Coudol, Experimental study of vortex emission behind bluff obstacles in a gas liquid vertical two-phase flow, International Journal of Multiphase Flow 8 (5) (1982) 475-490.

[17] R. Violette, M. J. Pettigrew, N. W. Mureithi, Fluidelastic instability of an array of tubes preferentially flexible in the flow direction subjected to two-phase cross flow, Journal of Pressure Vessel Technology 128 (1) (2006) 148-159.

[18] C. Monetter, M. J. Pettigrew, Fluidelastic instability of flexible tubes subjected to two-phase internal flow, Journal of Fluids and Structures 19 (2004) 943-956. 
[19] G. Tryggvason, R. Scardovelli, S. Zaleski, Direct numerical simulations of gas-liquid multiphase flows, Cambridge University Press, 2011.

[20] J. L. Riverin, M. J. Pettigrew, Vibration excitation forces due to two-phase flow in piping elements, Journal of Pressure Vessel Technology 129 (1) (2007) 7-13.

[21] D. G. Gorman, M. Liu, The effect of annular two-phase flow upon the natural frequencies of a pipe, Engineering Structures 20 (1998) 726-731.

[22] S. Dong, J. Shen, A time-stepping scheme involving constant coefficient matrices for phase field simulations of two-phase incompressible flows with large density ratios, Journal of Computational Physics 231 (2012) 5788-5804.

[23] S. Dong, On imposing dynamic contact-angle boundary conditions for wall-bounded liquid-gas flows, Computer Methods in Applied Mechanics and Engineering 247-248 (2012) 179-200.

[24] V. E. Badalassi, H. D. Ceniceros, S. Banerjee, Computation of multiphase systems with phase field models, Journal of Computational Physics 190 (2) (2003) 371-397.

[25] H. D. Ceniceros, R. L. Nós, A. M. Roma, Three-dimensional, fully adaptive simulations of phase-field fluid models, Journal of Computational Physics 229 (17) (2010) 61356155.

[26] D. Jacqmin, Calculation of two-phase Navier-Stokes flows using phase-field modeling, Journal of Computational Physics 155 (1) (1999) 96-127. 
[27] D. Anderson, G. B. McFadden, A. Wheeler, Diffuse-interface methods in fluid mechanics, Annual Review of Fluid Mechanics 30 (1) (1998) 139-165.

[28] H. Ding, P. D. Spelt, C. Shu, Diffuse interface model for incompressible two-phase flows with large density ratios, Journal of Computational Physics 226 (2) (2007) 2078-2095.

[29] D. Gerlach, G. Tomar, G. Biswas, F. Durst, Comparison of volume-of-fluid methods for surface tension-dominant two-phase flows, International Journal of Heat and Mass Transfer 49 (3-4) (2006) 740-754.

[30] C. S. Wu, D. L. Young, H. C. Wu, Simulations of multidimensional interfacial flows by an improved volume-of-fluid method, International Journal of Heat and Mass Transfer 60 (2013) 739-755.

[31] Y. Tsui, S. Lin, Y. Lai, F. Wu, Phase change calculations for film boiling flows, International Journal of Heat and Mass Transfer 70 (2014) 745-757.

[32] I. Chakraborty, G. Biswas, P. S. Ghoshdastidar, A coupled level-set and volume-of-fluid method for the buoyant rise of gas bubbles in liquids, International Journal of Heat and Mass Transfer 58 (1-2) (2013) 240-259.

[33] B. Ray, G. Biswas, A. Sharma, Bubble pinch-off and scaling during liquid drop impact on liquid pool, Physics of Fluids 24 (2012) 082108-1 - 082018-11.

[34] A. Criscione, D. Kintea, Z. Tukovic, S. Jakirlic, I. V. Roisman, C. Tropea, Crystallization of supercooled water: A level-set-based modeling of the dendrite tip velocity, International Journal of Heat and Mass Transfer 66 (2013) 830-837. 
[35] C. A. Fletcher, Computational Galerkin methods, 1st Edition, Springer Berlin Heidelberg, 1984.

[36] G. Guidoboni, R. Glowinski, N. Cavallini, S. Canic, Stable loosely-coupled-type algorithm for fluid structure interaction in blood flow, Journal of Computational Physics 228 (2009) 6916-6937.

[37] W. F. Noh, Cel: A time-dependent, two-space-dimensional, coupled Eulerian-Lagrange code, Tech. rep., Lawrence Radiation Lab., Univ. of California, Livermore (1963).

[38] C. W. Hirt, A. A. Amsden, J. L. Cook, An arbitrary Lagrangian-Eulerian computing method for all flow speeds, Journal of Computational Physics 14 (3) (1974) 227-253.

[39] J. G. Trulio, Theory and structure of the AFTON codes, Tech. rep., DTIC Document (1966).

[40] J. Donea, S. Giuliani, J. P. Halleux, An arbitrary Lagrangian-Eulerian finite element method for transient dynamic fluid-structure interactions, Computer Methods in Applied Mechanics and Engineering 33 (1) (1982) 689-723.

[41] P. Sun, J. Xu, L. Zhang, Full Eulerian finite element method of a phase field model for fluid-structure interaction problem, Computers \& Fluids 90 (2014) 1-8.

[42] T. J. Hughes, W. K. Liu, T. K. Zimmermann, Lagrangian-Eulerian finite element formulation for incompressible viscous flows, Computer Methods in Applied Mechanics and Engineering 29 (3) (1981) 329-349. 
[43] Y. Yu, B. Hyoungsu, G. E. Karniadakis, Generalized fictitious methods for fluidstructure interactions: analysis and simulations, Journal of Computational Physics 245 (2013) 317-346.

[44] G. E. Karniadakis, M. Israeli, S. Orszag, High-order splitting methods for the incompressible Navier-Stokes equations, Journal of Computational Physics 97 (2) (1991) 414443.

[45] H. Baek, G. E. Karniadakis, A convergence study of a new partitioned fluid-structure interaction algorithm based on fictitious mass and damping, Journal of Computational Physics 231 (2) (2012) 629-652.

[46] Y. Yu, H. Baek, M. L. Bittencourt, G. E. Karniadakis, Mixed spectral/hp element formulation for nonlinear elasticity, Computer Methods in Applied Mechanics and Engineering 213 (2012) 42-57.

[47] X. Zheng, H. Babaee, S. Dong, C. Chryssostomidis, G. E. Karniadakis, A phase-field method for 3D simulation of two-phase heat transfer, International Journal of Heat and Mass Transfer 82 (2015) 282-298.

[48] J. Towns, T. Cockerill, M. Dahan, I. Foster, K. Gaither, A. Grimshaw, V. Hazlewood, S. Lathrop, D. Lifka, G. D. Peterson, R. Roskies, J. R. Scott, N. Wilkins-Diehr, Xsede: Accelerating scientific discovery, Computing in Science \& Engineering 16 (2014) 62-74 doi:10.1109/MCSE.2014.80.

[49] G. Karniadakis, S. Sherwin, Spectral/hp element methods for computational fluid dynamics, Oxford University Press, 2013. 
[50] E. Nogueira, R. Cotta, R. Janeiro, Heat transfer solutions in laminar co-current flow of immiscible liquids, Warme-und Stoffubertragung 25 (1990) 361-367.

George Karniadakis received his S.M. (1984) and Ph.D. (1987) from

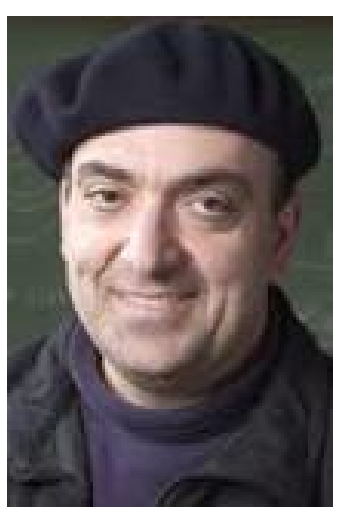

Massachusetts Institute of Technology. He was appointed Lecturer in the Department of Mechanical Engineering at MIT in 1987 and subsequently he joined the Center for Turbulence Research at Stanford / Nasa Ames. He joined Princeton University as Assistant Professor in the Department of Mechanical and Aerospace Engineering and as Associate Faculty in the Program of Applied and Computational Mathematics. He was a Visiting Professor at Caltech (1993) in the Aeronautics Department. He joined Brown University as Associate Professor of Applied Mathematics in the Center for Fluid Mechanics on January 1, 1994.

Xiaoning Zheng received her Ph.D (2012) from Purdue University.

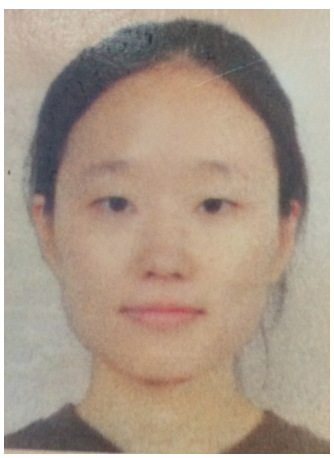
She worked as a postdoctoral associate in the Seagrant program of Department of Mechanical Engineering at Massachusetts Institute of Technology from 2013 to 2015. Now she joins in Applied Mathematics in the Center for Fluid Mechanics in Brown university and conducts research in computational mathematics. Her work on computational fluid dynam- 
ics includes simulation of turbulence, high-order numerical algorithm development and implementation, multi-phase flow modeling and simulation, heat transfer modeling and simulation and fluid-structure interaction. 